\title{
Calfatage d'or et éternité (Botel Tobago, Taiwan)
}

Gold caulking and eternity (Botel Tobago, Taiwan)

Calfateo de oro y eternidad (Botel Tobago, Taiwan)

\section{Véronique Arnaud}

\section{(2) OpenEdition}

\section{Journals}

Édition électronique

URL : https://journals.openedition.org/tc/293

DOI : $10.4000 /$ tc. 293

ISSN : 1952-420X

Éditeur

Éditions de l'EHESS

Édition imprimée

Date de publication : 1 janvier 2001

Pagination : 255-285

ISSN : 0248-6016

Référence électronique

Véronique Arnaud, «Calfatage d'or et éternité (Botel Tobago, Taiwan) », Techniques \& Culture [En ligne], 35-36 | 2001, mis en ligne le 07 septembre 2012, consulté le 29 septembre 2022. URL : http://

journals.openedition.org/tc/293 ; DOI : https://doi.org/10.4000/tc.293

Ce document a été généré automatiquement le 29 septembre 2022

Tous droits réservés 


\title{
Calfatage d'or et éternité (Botel Tobago, Taiwan)
}

\author{
Gold caulking and eternity (Botel Tobago, Taiwan) \\ Calfateo de oro y eternidad (Botel Tobago, Taiwan)
}

Véronique Arnaud

1 Installés à Botel Tobago au large des côtes sud-est de Taiwan, les Yami forment une population d'environ deux mille huit cent âmes. Ils parlent une langue austronésienne proche de l'ivatan et de l'itbayat des îles Batan (Philippines) où résident des groupes qui leur sont apparentés. Leur système de filiation bilatéral attache cependant plus d'importance à la ligne paternelle. Bien que les Yami soient christianisés, le panthéon et le système de pensée traditionnels jouent un rôle déterminant dans leur vie quotidienne. Pêcheurs mais aussi agriculteurs, ils pratiquent la culture irriguée du taro, la culture sèche de la patate douce et du millet, et l'arboriculture fruitière.

2 Dans un article paru en 1995, j'avais étudié la répartition des bateaux et les méthodes de pêche utilisées au fil des trois saisons du calendrier yami : «la fin de la saison de pêche » (teiteika, juillet-décembre), la saison froide, « où l'on reste sur place » (amyan, décembre-mars) et la «saison de pêche des poissons volants» (rayun, mars-juillet). J'avais ainsi répertorié plus d'une vingtaine de techniques de pêche différentes adaptées aux deux types d'embarcation : la petite et la grande pirogue.

Les petites pirogues servent presque toute l'année et sont la propriété des rameurs. Les grandes pirogues portent le patronyme du lignage dont elles dépendent: elles appartiennent à des "groupes de pêche" (asa cicipunan) fondés sur le groupe de filiation patrilinéaire et composés de «huit ou dix hommes » (sangarnan du cinedkeran) selon la taille du bateau. Conçues comme le bateau de pêche des poissons volants (exocets), elles ne sont utilisées que pendant quatre mois (de mars à juillet²), lorsque passent les exocets migrateurs venus du Sud. De mars à avril, la pêche aux poissons volants se pratique la nuit à l'aide de torches et de grandes épuisettes: elle est peu productive. Les lunaisons, Papataw et pipilapila (mai-juin) désignées comme «le temps de pêche des exocets » fournissent en deux mois $70 \%$ des prises totales de la saison. 
C'est une période de "pêche intense à la senne " (meitawar) qui s'effectue de jour au "lieu de pêche des poissons volants", lieu propre à chaque village, et rassemble un grand nombre de participants : membres de l'association de pêche, membres du groupe détenteur du filet, et tous les nageurs venus aider. La lunaison est également associée à la pêche en petite pirogue de jour (à la dorade coryphène) et de nuit (aux poissons de grosse taille comme le thon). En dehors de la saison de pêche, les grandes pirogues restent à l'abri dans des constructions en longueur à murs de galets et toit de chaume situées près du port.

M'appuyant sur des données de terrain récoltées depuis une vingtaine d'années, j'analyserai ici la construction des grandes pirogues assemblées. Elle se déroule en trois phases: la préparation d'une durée de deux à trois ans jusqu'à maturité des taros accumulés pour le rituel de lancement; la construction proprement dite, désignée par le terme de "travail» (meivarei), "le travail (accompli pour) la grande pirogue» (meivarei su cinedkeran) par les dix membres du groupe de pêche, unissant leurs forces dans cet ouvrage; puis le rituel de lancement où l'on se propose de «nourrir » le bateau et de lui « donner vie, corps et esprit ».

\section{Type de pirogues et répartition géographique}

5 À Botel Tobago, les grandes pirogues (cinedkeran) mesurent environ sept mètres de long et les petites (tatala) trois mètres. Elles sont de forme et de conception identiques avec des proues et des poupes très élancées qui constituent les deux extrémités de la quille sur laquelle viennent s'assembler les bordés faits de planches ${ }^{3}$ (fig. 1).

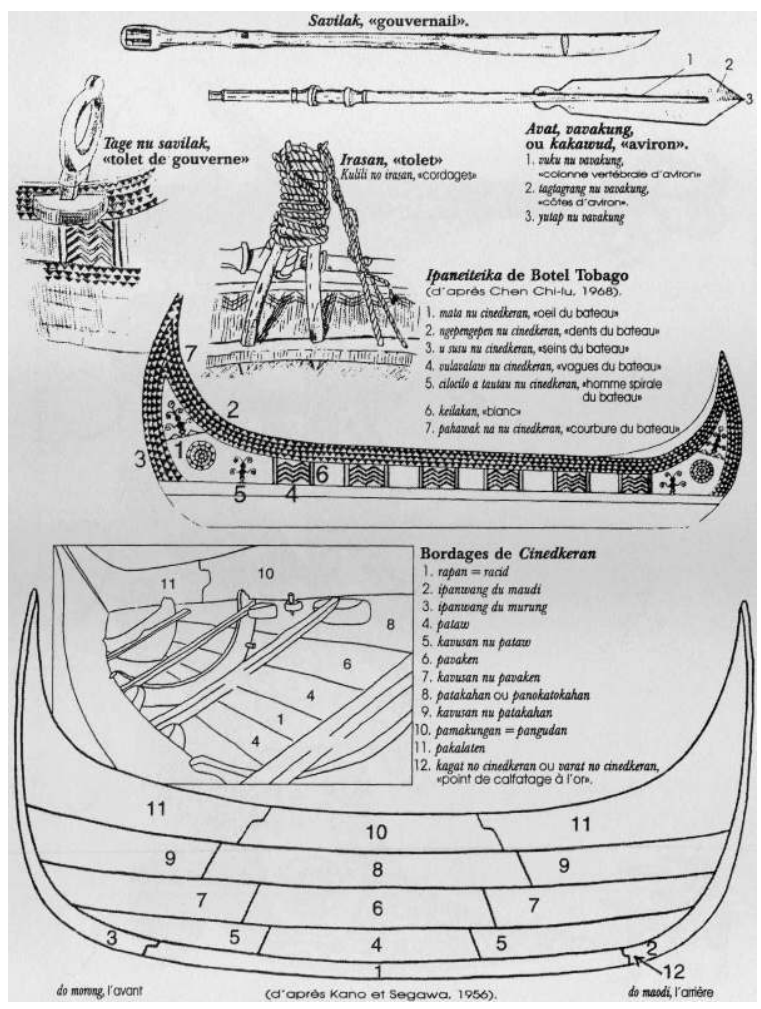

Figure 1. Cinedkeran de Botel Tobago 


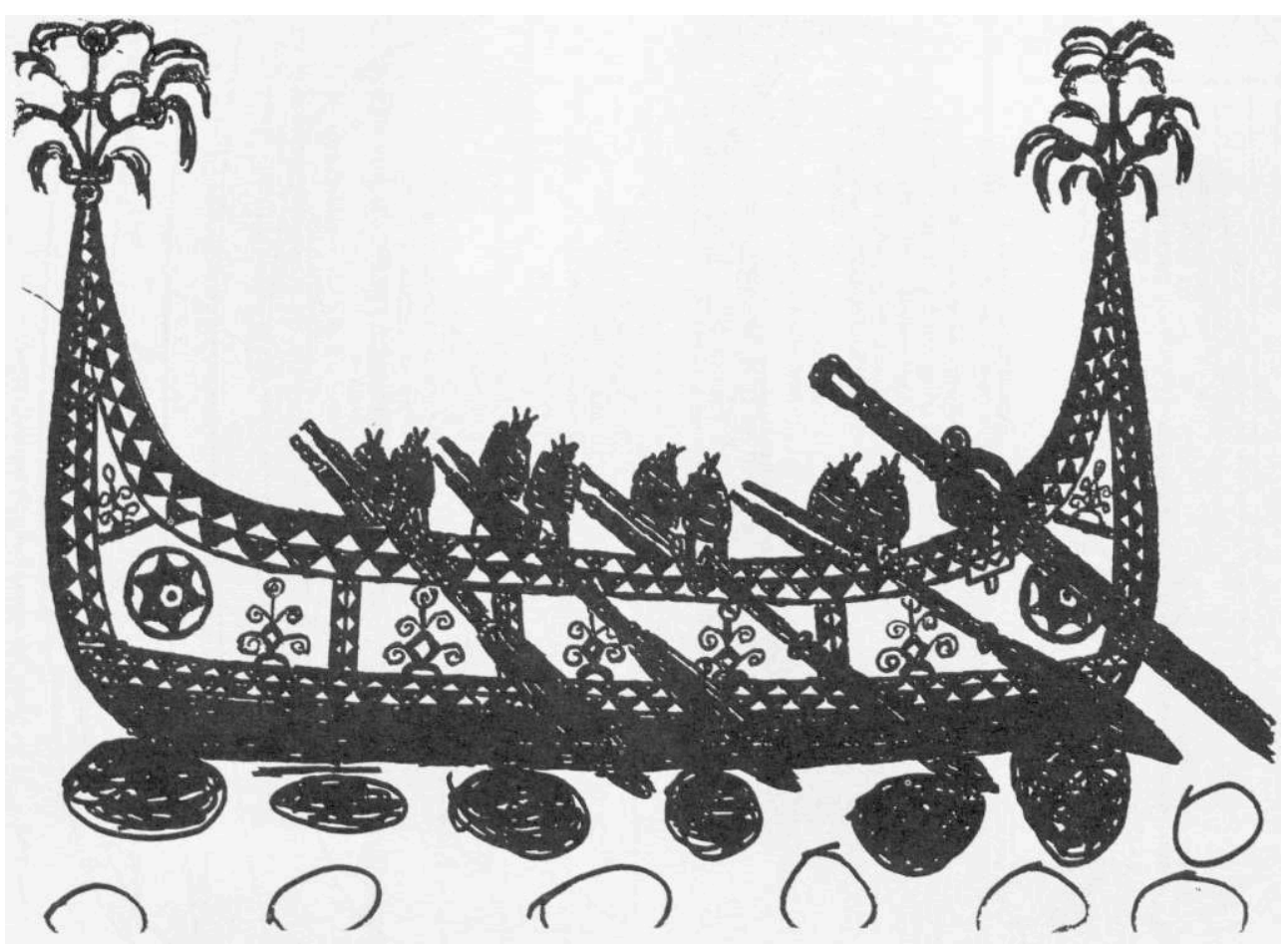

Figure 2. Grande pirogue « quatre avirons » (Si-Ngarapad, Jiraralei, 1974)

Comme les grandes maisons à quatre portes couvertes de "quatre couches de chaume » (apat a inapan a vucid), la coque de la grande pirogue présente "quatre couches de bordés" (apat a inapan), chacune composée de trois bordés, formant un total de $(2 \times 12)=24$ pièces de bois assemblées par des chevilles. Les couches sont montées successivement à partir de la "quille " (rapan ou racid), des "extrémités de poupe " (ipanwang du maudi) et de " proue » (ipanwang du murung). On commence par la « couche du bas» (kavusan nu pataw, pataw du teirawem, kavusan nu pataw), puis on monte la "deuxième couche" (kavusan nu pavaken, pavaken, kavusan nu pavaken), la «troisième couche" (kavusan nu panukatukahan, panukatukahan ou patakahan, kavusan nu panukatukahan) et enfin la "couche du haut" (pakalaten du murung, pamakungan ou pangawudan, pakalaten du maudi). Le dernier bordé est terminé en plat-bord. Des bancs de nage sont fixés le long des deux bords qui entourent une cavité centrale servant de compartiment pour les poissons à l'avant comme à l'arrière, et au centre, de rangement pour le matériel. Le fond et les flancs du bateau sont renforcés par des varangues et des membrures posées après la mise en place des bordés. Des lisses transversales, fixées sur les membrures, soutiennent l'ensemble. L'écartement des extrémités du bateau -proue et poupe- est maintenu par une plaque de bois triangulaire. À la base de la " proue » (du murung), se trouve une emplanture (peivilaviladan), destinée au mât et à la voile, pour la navigation en haute mer. À la plus haute extrémité de la poupe (du maudi), est prévu un support pour les torches servant lors de la pêche aux poissons volants ; à sa base, un « abri pour le feu » (pangayan su apui) est ménagé.

7 Les petites pirogues peuvent embarquer un ou deux hommes et sont munies d'avirons à chaque bord. On les appelle peikatangyan, "où poser son derrière ", pour une personne et pour deux, peikavangan ou peiciriciringan, «le bateau où l'on peut faire la conversation ». Les grandes pirogues sont dirigées par un chef de pirogue qui fait office de barreur. Chaque rameur y occupe une place précise. Ces pirogues sont armées en 
pointe avec un aviron (d'une longueur approximative de deux mètres) par rameur. À la manière des maisons d'habitation qui se distinguent par le nombre de portes, les grandes pirogues sont désignées par le nombre d'avirons disposés sur chaque bord : il y a la «trois» -ou «trois avirons »- qui porte six hommes, la «quatre » qui en porte huit (fig. 2) et la " cinq", qui en porte dix. Les avirons sont solidement fixés entre des tolets -à l'aide de cordages - et celui de queue, le gouvernail (d'environ cinq mètres), est engagé dans un tolet de gouverne circulaire qui permet le maniement de l'embarcation. Ils sont gravés de motifs anthropomorphes figurant «la colonne vertébrale de l'aviron » (vuku nu vavakung), et « ses côtes » (tagtagrang nu vavakung). Si les bordés sont gravés de motifs décoratifs, la grande pirogue est appelée ipaneiteika; quand elle n'est pas décorée, elle porte le nom d'ipeiraun. Parmi les motifs qui permettent d'identifier les groupes de pêche des différents lignages, on distingue des "rangées d'hommes de grande pirogue » (malagilagit a tautau nu cinedkeran) et des "vagues de grande pirogue » (malagilagit a vulavulaw nu cinedkeran) (photo 1). La grande pirogue a quelque chose d'« humain» puisqu'elle comporte des "yeux» (mata nu cinedkeran) à l'avant et à l'arrière du bateau, des "dents " (ngepengepen nu cinedkeran), en chaînes de triangles noirs et blancs sut les bordés supérieurs (photo 2), et des "seins" (u susu nu cinedkeran) en forme de losanges dans la partie antérieure de la poupe et de la proue qui lui confèrent une image féminine. Tous ces motifs sont peints en rouge et noir sur fond blanc (keilakan) ${ }^{4}$. À l'extrémité de la poupe et de la proue sont fixées des ornements décorés de figures humaines et de plumes de coq appelés les "proues de bateau» (murung nu tatala) ou les "plumes de grande pirogue » (ngayi nu cinedkeran) (photo 3).

De par son armement conçu pour résister aux assauts du vent, la pirogue servit sans doute, jusqu'au milieu du XVIIe, à des voyages au long cours et à des migrations. Selon Kano (1931), ce type de bateau a été observé en divers endroits d'Asie du Sud-Est insulaire, de la presqu'île de Malacca, en passant pat les Célèbes, les Moluques, la met de Banda et les côtes de la Nouvelle Guinée. Aux îles Salomon orientales en Mélanésie, existaient au début du siècle des bateaux de guerre très semblables, avec une poupe et une proue très hautes, ornées de figures anthropomorphes (Fox 1908). Le rouge, le noir et le blanc étaient également les couleurs traditionnellement employées (Wood-ford 1909). Plus près de Botel Tobago, sut les îles Batan des Philippines, on observe des techniques de construction, des méthodes de pêche et des rituels similaires. Le vocabulaire ibatan-espagnol des missionnaires Dominicains (1933), comprend deux termes apparentés au yami: tataya, "un petit canot» et chinedkeran, "une grande embarcation propre à l'île d'Itbayat ${ }^{5}$ ». Cihnedkeran désigne en itbayat une embarcation avec trois ou cinq avirons sur chaque bord, pouvant emporter six ou dix hommes d'équipage et dix passagers et servant à se déplacer entre les îles. Selon la tradition des Yami, les bateaux de grande taille auraient disparu après la rupture des relations avec les Ivatan et les Itbayat. On retrouve un vocabulaire commun: "proue» (itbayat murung, yami murung), "poupe » (itb. mawdi, yami maudi), « quille» (itb. rachid, yami racid), etc. (Yamada 1966-67). 


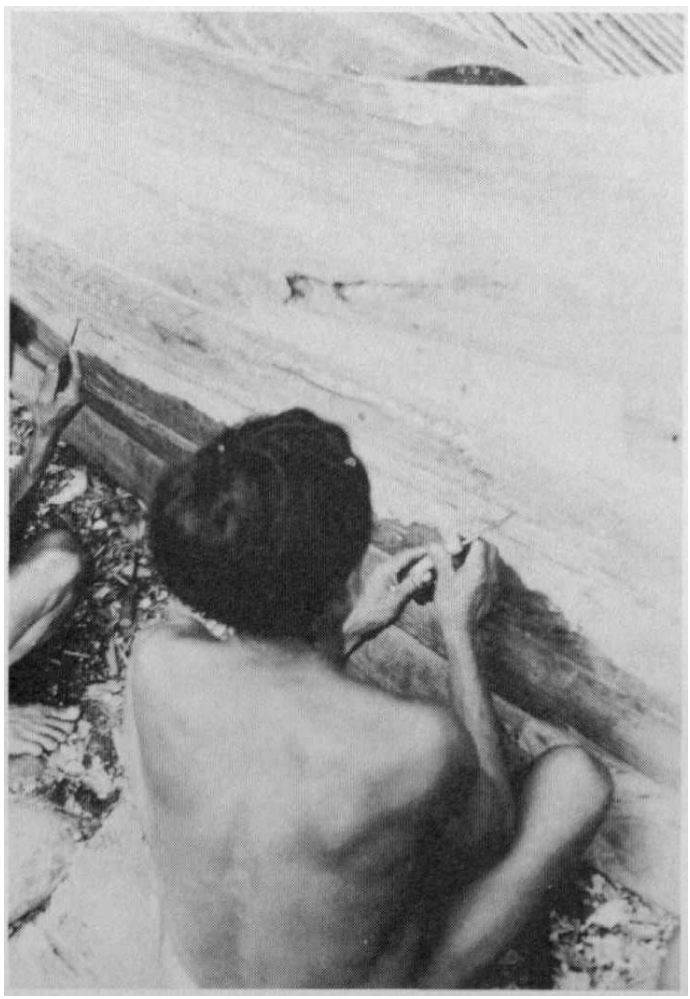

Photo 1. Gravure des « vagues » du bateau (Jiranumeilek, 1977)

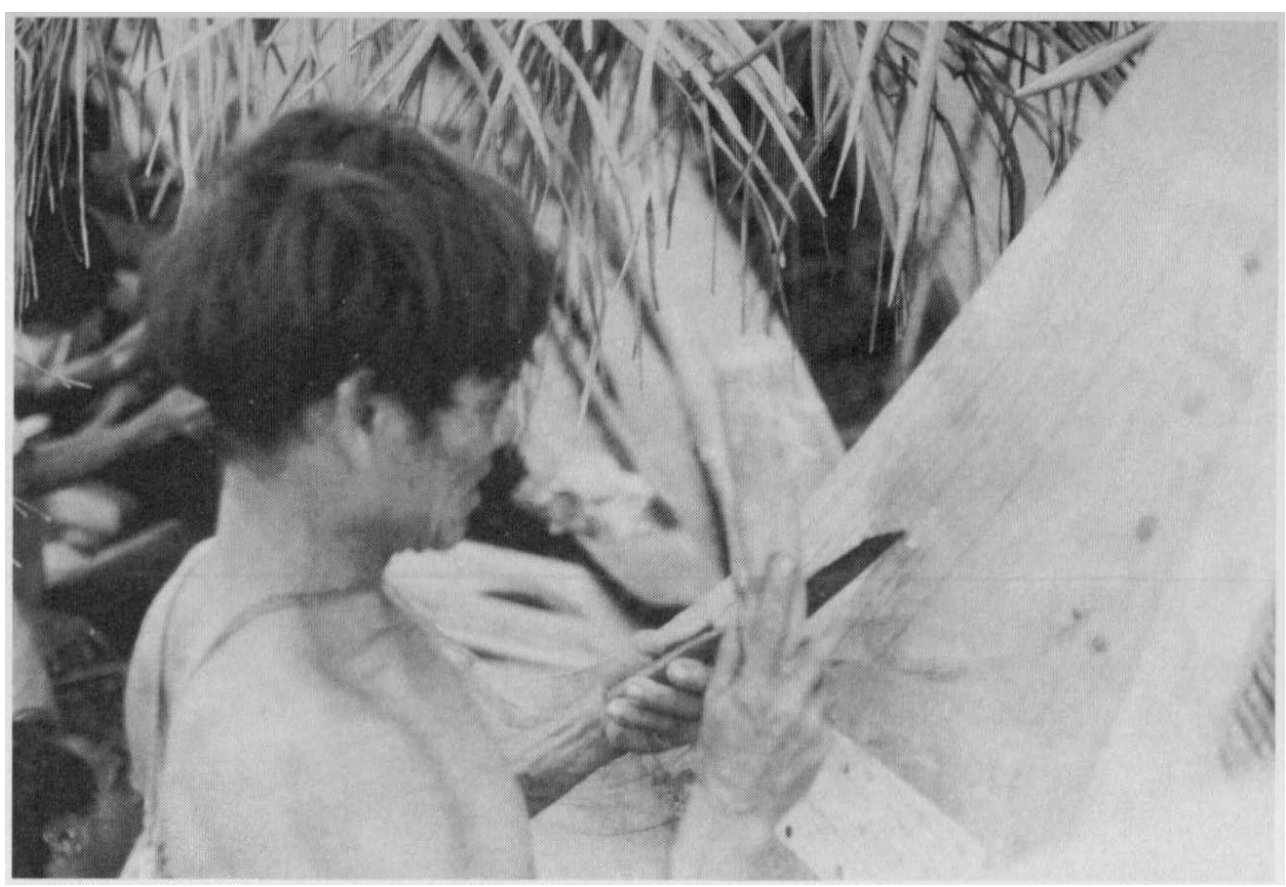

Photos 2. Gravure des « dents » du bateau (Jiranumeilek, 1977) 


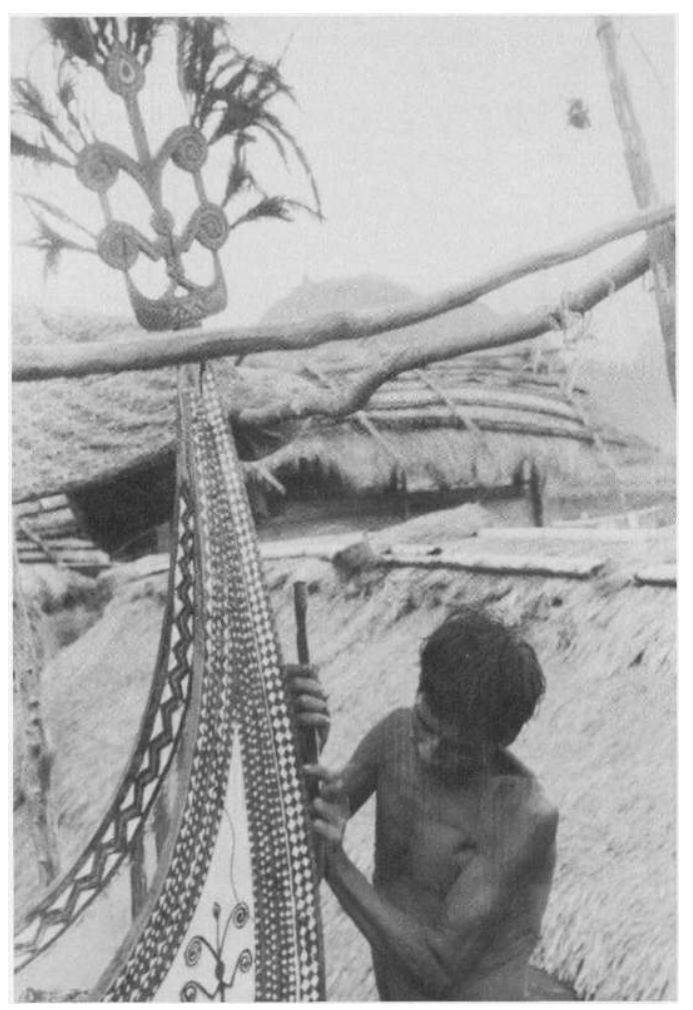

Photo 3. Gravure de l'élancée de poupe (Jiranumeilek, 1977)

Les techniques de construction sont similaires : assemblage des différentes pièces de bois sur la quille qui soutient toute la structure et se prolonge à l'arrière et à l'avant ; calfatage avec de «l'étoupe des racines" de l'arbre varuk ${ }^{6}$ (itb. varuk, yami varuk); ajustement des planches dont les jointures sont enduites d'argile (itb. vurilaw, yami vurilu); outillage commun : essentiellement la hache pour tailler et le ciseau à bois pour aplanir; enfin, les espèces de bois employées pour la confection des bordés sont les mêmes.

\section{Le projet de construction}

La grande pirogue étant réservée à la pêche aux poissons volants, la décision de la construire doit être prise le jour même de l'ouverture. Un rituel est accompli vers la fin du mois de février : on "fait le port " (meivanuwa) en vue d'attirer les poissons jusqu'au lieu de pêche du village (Arnaud 1992). Les hommes portent à cette occasion leur heaume d'argent, leur pectoral d'or, et les femmes leur long collier d'agathe et de cornaline : «Non, je ne reste pas dans ma maison », chante Siapen-Jabag du lignage DuChemin, responsable des rites d'ouverture de la pêche (Jiraralei 1974), «je me dresse sur ma terrasse pavée où j'arbore mon heaume d'argent et mon pectoral d'or. Brillant de mille éclats, nos parures se verront de loin. Et les poissons diront : que c'est beau, et ils approcheront pour voir ». Les parures peuvent aussi être suspendues sur les perches de séchage des poissons.

11 Autrefois, les ancêtres du lignage Du-Chemin (Sira Du-Rarahan) qui détenaient la responsabilité des rites d'ouverture, dont Siapen-Jabag a hérité, avaient coutume de tendre leurs plaques d'or vers le ciel pour inviter les divinités du haut à guider les poissons volants vers leur territoire de pêche. Le responsable, un «ancien riche et 
prospère » (meinakem a rarake), indiquait les places respectives des grandes pirogues sur le port, et au moment d'armer les avirons pour la première fois, jetait à la mer cinq «perles bleues » (marapunei) ${ }^{7}$ et une lamelle d'or de la taille d'un ongle. De nos jours, à Botel Tobago, on ne jette plus ni perles bleues ni or à la mer mais seulement le sang ${ }^{8}$ des animaux sacrifiés ${ }^{9}$ : généralement celui d'un porcelet -ou d'un coq- saigné en pleine mer dans la grande pirogue de l'association ouvrant la pêche, la plus belle et la plus récente que l'on place, pour la valoriser, sur la droite du port. L'animal levé vers le ciel est secoué en direction de l'horizon pendant que l'on appelle les exocets. Les galets du rivage sont également enduits de sang pour attirer les poissons. Ceux qui n'ont pas de sang peuvent en échanger contre de l'or, des perles bleues ou de la monnaie de Taiwan. Ils en emplissent de petits tubes de bambou qu'ils suspendront par la suite dans leur bateau ou dans leurs champs pour favoriser l'abondance de la pêche et des récoltes ${ }^{10}$.

Cette offrande permet au responsable de l'ouverture de la pêche, considéré comme un chamane, de lever les interdits et d'obtenir des poissons volants. À l'aide d'une longue spatule à gruau de millet ou « rame à oublier " (kakawu), celui-ci a le pouvoir de «faire oublier » (makawu) ou de « renverser la pensée » (peitaliyurung su nakem) des poissons en cas de transgression d'un interdit par un membre du village ${ }^{11}$. Il peut ainsi faire tonner la foudre ou lever la tempête contre les transgresseurs, "faire oublier " les poissons volants et obtenir qu'ils reviennent. Il tente de "s'oublier » lui-même pour que son "âme de gauche voyageuse » divague vers les sphères célestes et intercède auprès des Hommes du Haut en faveur des contrevenants pour qu'ils guident les exocets vers leur territoire maritime.

La décision de construction d'une grande pirogue doit être tenue secrète afin de ne pas attirer l'attention des «morts errants» (anitu), considérés comme une menace pour le "groupe constructeur » et pour son bateau, car les Yami pensent que les anitu aiment beaucoup les grandes pirogues décorées et sont toujours prêts s'en emparer. Il faut déjouer leurs pièges car ils cherchent à faire le travail à la place des vivants et à les affaiblir en leur "amollissant les veines ${ }^{12} \mathrm{Il}$ ne faut donc parler à personne des préparatifs, et répondre par la négative si par hasard on vous interroge à ce sujet, car les hommes ont besoin de toutes leurs forces pour venir à bout des durs travaux qui leur incomberont durant ces deux ou trois années de préparation jusqu'à la maturité des taros.

14 C'est au moment de l'« appel rituel des poissons volants sur le port » que se forme le "groupe constructeur » d'une grande pirogue, qui deviendra le futur groupe de pêche du lignage dirigé par son responsable et barreur. Les hommes se rassemblent chez leur barreur pour discuter du projet. Lorsqu'il conçoit ce projet, le groupe constructeur fait le compte des offrandes susceptibles d'être rassemblées pour la cérémonie de lancement: la quantité de taros ordinaires et de grands taros pour lesquels il sera nécessaire d'ouvrir de nouvelles tarodières quand viendra la «fin de la saison de pêche » (de juillet à décembre), le nombre de porcs et de chèvres, propres à chacun, qui seront engraissés ou achetés, de "saupes grises ${ }^{13}$ ou autres poissons de bon augure afin de les suspendre sur le bateau ; l'épaisseur, enfin, de la plaque en or qui constituait autrefois l'offrande du chef de pirogue et était glissée entre les joints du bordage. 


\section{Le bois d'œuvre}

La construction des bateaux a lieu en saison de « fin de pêche des poissons volants » à la « lunaison d'or » (juin) ou à la "bonne lunaison » (juillet) -en début d'année lunaire. Elle commence par la recherche collective en montagne des arbres destinés aux bois d'œuvre, jaugés sur pied et marqués d'un signe de reconnaissance du lignage, qui doivent ensuite être acceptés par l'ensemble du groupe. Reconnaître les arbres à couper est un travail difficile car les vingt-quatre planches du bordage doivent être faites, respectivement, d'essences végétales différentes. Pour les quatre bordés supérieurs latéraux de la poupe (pakalaten du maudi) et de la proue (pakalaten du murung), on emploie, pour leur courbure naturelle, les larges racines adventives du "longanier " ${ }^{14}$, de l'arbre à pain ${ }^{15}$ ou d'autres arbres de la forêt présentant les mêmes caractéristiques ${ }^{16}$. Lors du travail en forêt dense, considérée comme un repaire d'anitu, les bûcherons prennent la précaution d'offrir du millet et de la viande de porc et de chèvre aux "esprits des morts». Ainsi, les hommes ne risqueront pas de se faire "pincer» (= mourir), et le transport du bois d'œuvre se fera plus facilement. Il est parfois nécessaire d'entonner " un chant efficace de la forêt » (karusan du kahasan) pour avertir les «êtres sans visage » qui rôdent tout autour de la construction en cours d'un «bateau décoré en proue »:

«Qui n'a plus de force à abattre les arbres - Qui voit ses veines ramollies - Ne comprend pas ce qui l'exténue - Étant très fort par nature à abattre les arbres Qui serait l'eau, qui serait le "sureau" - Légers entre ses mains à débiter en morceaux. » (Arnaud 1979: 144-147).

Cette déclaration vise à « durcir le réseau des veines » et à redonner la "force vitale » permettant à l'artisan de poursuivre son travail. Les pièces de bois, ainsi dégagées des «morts » qui pesaient sur elles, se font plus légères entre les mains des constructeurs.

\section{La poupe et la proue}

17 Les bordés sont façonnés à mesure que l'on construit le bateau en commençant par les extrémités de poupe et de proue qui viennent en prolongement de la quille et font la splendeur des embarcations yami. Le façonnage de ces deux pièces est soumis à un interdit de pêche pour les hommes qui peuvent à cette occasion apporter des soins aux taros, une tâche habituellement réservée aux femmes. Tout au long du "travail », les dix hommes tentent «de durcir et de fortifier » leur "construction en cours " par l'utilisation de bois « durs et forts » pour les bordés, par des rites de longévité dédiés aux différentes pièces de bois, par l'apposition d'or, et par l'onction de sang qui « donnent peu à peu la vie » au bateau.

18 L'« extrémité de la poupe " (ipanwang du maudi), valorisée par rapport à celle de la proue, est fabriquée en premier lieu ; puis vient l'« extrémité de la proue » (ipanwang du murung) (photo 4). Pour ces deux pièces, il importe d'employer des «bois durs" répertoriés dans la classification indigène comme ceux d'" arbres forts" tels que le "faux badamier $»^{17}$ ou d'autres " arbres forts de la forêt " (muuyat a kayu du kabasan) ${ }^{18}$. Une fois le terrain éclairci, les arbres sont abattus à la hache et leurs grumes débitées. La poupe et la proue sont façonnées sur place, toujours à la hache, puis amenées ensemble le jour même sur le lieu de construction situé dans le village, généralement un terre-plein devant la maison du barreur. 
Le lendemain, un «rite de longévité avec de l'or» (pabwebwesan nu ipanwang) est accompli : maintenues dressées, ces deux pièces de bois sont rapprochées, "mêlées l'une à l'autre et attachées solidement entre elles » (peikedkedan da nya a ipanwang), liées à jamais dans un avenir commun.

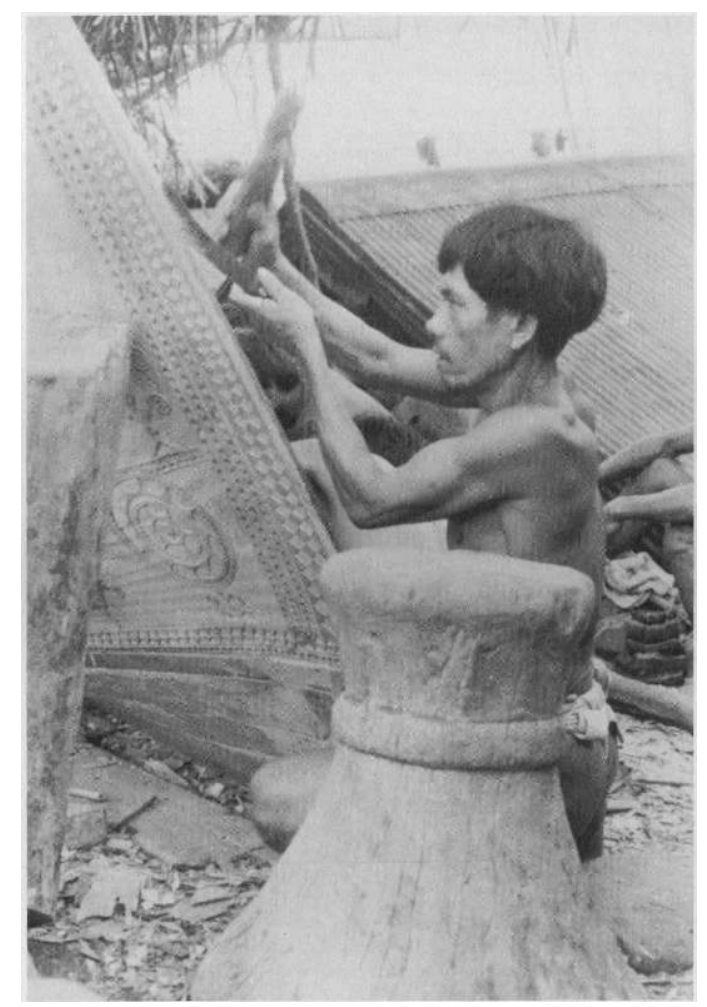

Photo 4. Gravure de l'élancée de proue (Jiranumeilek, 1977)

Le responsable de la grande pirogue du lignage les touche ensuite avec de l'or « porteur de longue vie » (ipanlallag), l'une après l'autre, en commençant par la poupe, et leur adresse une invocation pour les « célébrer » (tuytuynen):

«J'applique de l'or ${ }^{19}$ sur toi, une belle peau qui vient te fortifier, projection de poupe [ou projection de proue] de notre grande pirogue. Tu es le chef des bordés de notre grande pirogue, celle qui domine et soutient toutes les autres à la fois. Que cet or te donne la vie, que tu dures longtemps et demeures à jamais dans la clarté ! Par cet or, reste toujours dressée bien droite, reste toujours vive et pleine d'entrain! Qu'il nous fortifie nous aussi et que nous vivions à jamais dans la clarté. Issus, tous les dix, en droite ligne d'un même ancêtre, nous t'assemblons et te dressons bien droite également. Reste droite à jamais chère et honorée projection de poupe [ou chère et honorée projection de proue]! Comment pourrions-nous venir à bout de notre travail et trouver le repos si nous ne t'avions pas célébrée de la sorte ?»

Chacun des membres du groupe de pêche les touche à son tour avec de l'or pour leur «donner la vie » les pressant, à l'exemple de leur chef, de durer longtemps, de rester toujours bien droites et de vivre avec eux à jamais dans la clarté. Les « constructeurs de la pirogue " estiment que ce rite de longévité tend les pièces de bois plus légères qu'elles ne l'étaient lors de leur transport à travers la forêt et les pentes abruptes de la montagne. 


\section{La « quille du bateau »}

La quille, (rapan nu cinedkeran ou racid nu cinedkeran) façonnée après la poupe et la proue, requiert le sacrifice d'un porc dont l'homme de barre lui offrira le sang à son retour au village. En «enduisant la quille du bateau avec le sang du porc » (raralaen $u$ racid na nu rala nu vinyei), il l'exaltera de ces mots invoquant sa durabilité et la fertilité de la pêche :

«Toi, notre quille de grande pirogue sur laquelle s'appuient tous les bordés, puisses-tu vivre longtemps! Nous saignons ce porc à ton intention chère et honorée quille; chaque fois que tu sillonneras les mers, que son sang fasse venir à toi des poissons, de nombreux poissons! Puissent les hommes que tu portes ne pas revenir bredouilles et sachent en pêcher des multitudes. Qu'avec toi, ensemble, ils vivent très longtemps! »

\section{Les « couches de bordés »}

Une fois « rabotées au ciseau à bois » (taripsen nu kawar), les bordés sont assemblés les uns après les autres, puis à nouveau aplanis une fois montés. Pour que la coque soit symétrique, on tend un fil entre l'avant et l'arrière du bateau lors de l'assemblage. Sa largeur est déterminée par le nombre de doigts et un bâton est utilisé pour vérifier l'horizontalité. Le montage des bordés est suivi du démontage puis de la fabrication des "chevilles" (yurei) en bois de mûrier blanc (pasek, mûrier blanc, Morus alba, L., Moracée) d'environ quinze centimètres de long. Pour une grande pirogue, on compte plus de trois mille chevilles insérées tous les cinq centimètres dans des trous creusés dans les bordés à l'aide d'une gouge (paet). Les jointures des planches sont ensuite enduites d'argile puis calfatées à l'étoupe des racines de l'arbre varuk sur une épaisseur d'un centimètre environ. L'étoupe est maintenue par un lien d'abaca (avaka, Musa textilio, Née, Musacée) aux chevilles du bateau. Une fois la coque assemblée à l'aide d'une pierre servant de masse, on en consolide le fond par des membrures, puis la construction se termine par la gravure des bordés. À chaque phase, il convient de sacrifier un porc et d'oindre de sang le bateau tout en l'invoquant pour qu'il vive longtemps : au début du « montage de la couche des bordés du bas » (peitwatwangen), au commencement du «calfatage à l'étoupe » (tumbek su varuk, mamaruk su cinedkeran nu varuk), de «l'assemblage des bordés par des chevilles de bois» (tumbek su yurei) et du travail enfin de "gravure des motifs décoratifs » (mapatakatakad su vatek) sur les bords (photo 5). 


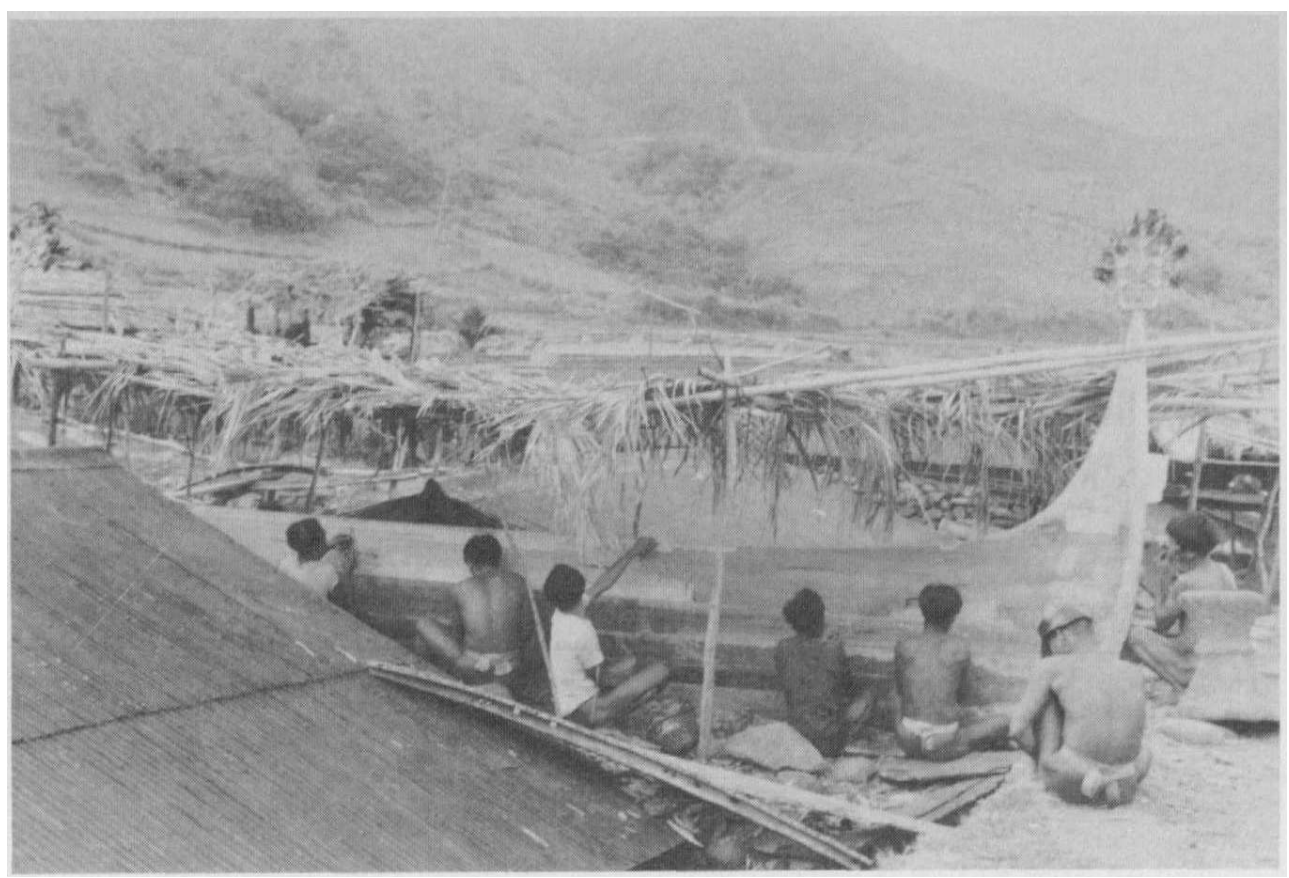

Photo 5. Début des gravures d'une grande pirogue (Jiranumeilek, 1977)

\section{Le calfatage à l'or}

Autrefois, avant l'exorcisme $\mathrm{du}$ bateau, l'«Arrière-grand-père du lignage constructeur ", appelé également l'« Arrière-grand-père-de-la-poupe » (Siapen-kutan Du-maudi), avait coutume d'insérer dans l'étoupe de calfatage, entre les jointures de la poupe et de la quille, une plaque d'or de la taille d'un ongle destinée à « durcir à jamais les veines » des bordés. Il était accompagné de sa femme, l'« Arrière-grand-mère-de-lapoupe " (Siapen-kutan Du-maudi) qui faisait sauter, en même temps que lui et de la base de la poupe, une « cheville de grosse taille en bois de mûrier blanc » nommée varat nu cinedkeran (asuwaten u kagat nu cinedkeran) ce qui signifie « le point de ralliement, le lieu où convergent les forces (de la grande pirogue) $»^{20}$

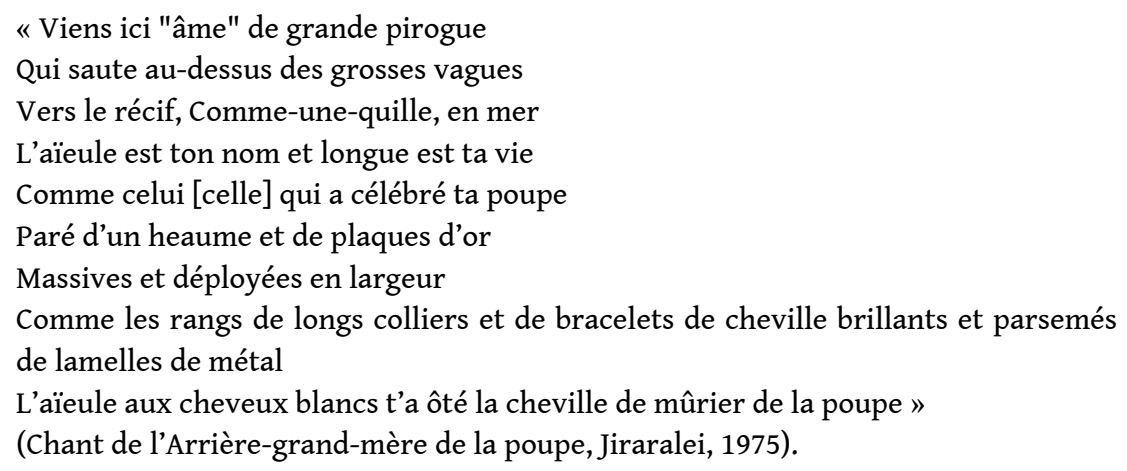

Le bateau ainsi "célébré par de l'or en poupe de bateau » (tuytuynen u nivarat da a cinedkeran), est, à l'exemple des Arrière-grands-parents-de-la-poupe, "promis à une longue destinée » (yapiya u lag na nu cinedkeran).

L'Arrière-grand-père-de-la-poupe entonnait alors le chant de calfatage ou le « chant de ralliement entre le métal d'or, la "belle peau", et la grande pirogue » (ianuud da su nivarat nu tametamek a piya su kulit da a cinedkeran) dont voici la traduction : 


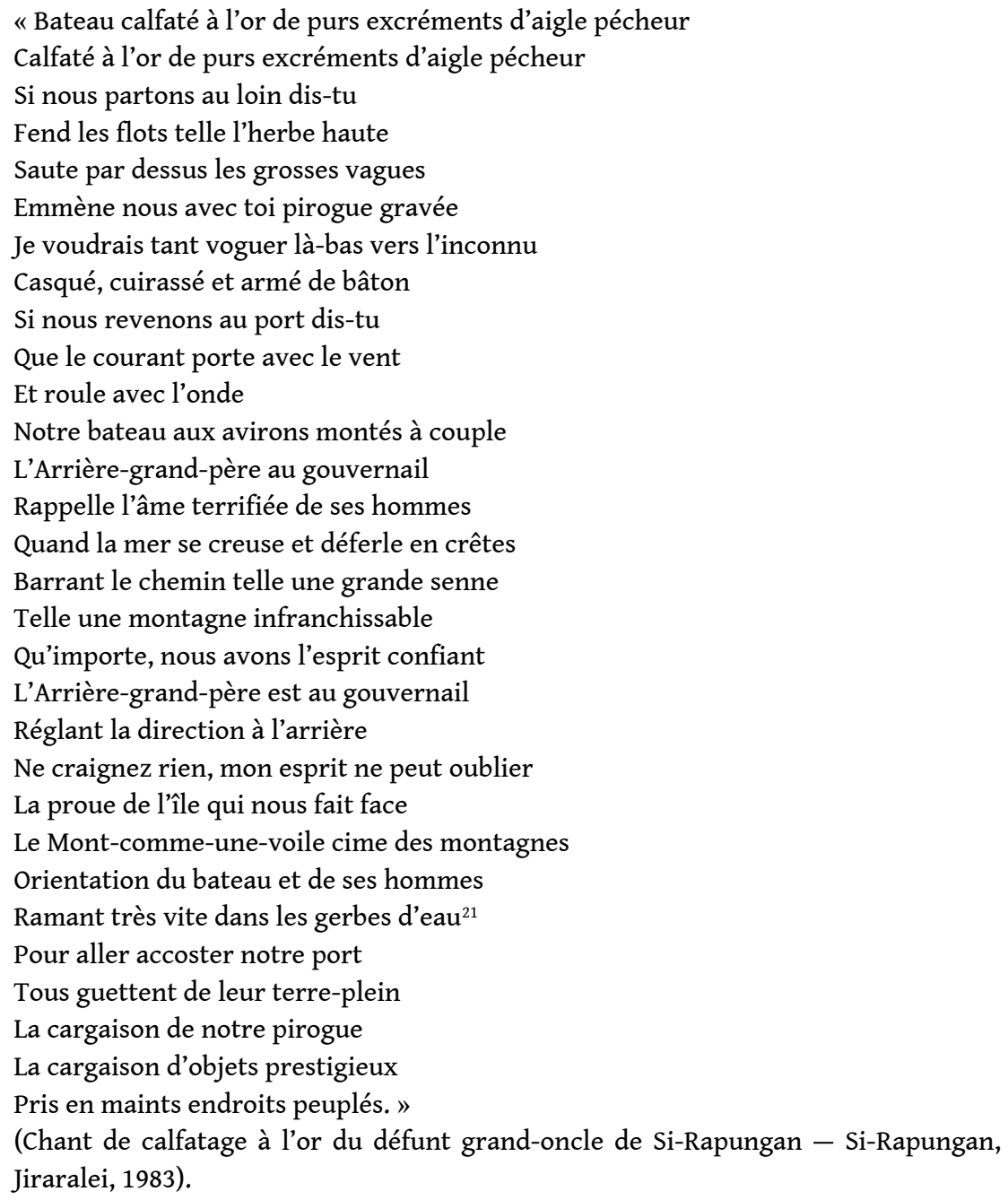

\section{La destinée de la grande pirogue calfatée d'or}

Il semble que la tradition de «calfater d'or les pirogues" (varaten nu cinedkeran nu tametamek) se soit perpétuée jusqu'il y a une trentaine d'années à Jiraralei. Un ancêtre célèbre du lignage De-bordure (Sira Du-seili), en la personne du grand-oncle défunt de mon informateur et ami Si-Rapungan, en aurait fait l'expérience lors de la construction de deux de leurs grandes pirogues. Dans la mythologie, l'aïeule ayant découvert les excréments d'aigle pécheur est issue, en ligne maternelle, du lignage De-bordure (akes Sira Du-seili) et c'est dans ce lignage que se sont transmis les récits, les chants et les pouvoirs de «calfater à l'excrément d'aigle pécheur » (ubut nu kangkang ni varat da su cinedkeran). Le grand-oncle défunt, qui en a hérité, a ainsi calfaté une «belle peau » de la taille d'un ongle au point de ralliement (entre la poupe et la quille, entre l'or et le bateau) de leur grande pirogue du lignage De-bordure. S'il se l'est permis lui aussi, c'est parce que les dix membres de son groupe de pêche appartenaient tous, en ligne paternelle, au " même lignage De-bordure » (asa su inawan Sira Du-seili). Ce qui était la norme autrefois dans la constitution des groupes de pêche en grande pirogue. Lorsque l'ancêtre a "calfaté leur pirogue à l'excrément d'aigle pécheur ", il a composé lui-même un chant à l'adresse de son bateau que son neveu a rappelé en 1983. 
par son pouvoir d'attraction sur les poissons, l'or est estimé favoriser la fertilité de la pêche. Lorsque les pêcheurs attrapent des poissons de grande taille, tels une grosse dorade coryphène ou un thon pat exemple, ils ont coutume de retour chez eux, de leur glisser dans la partie ventrale une plaque d'or tout en les invoquant de ces mots : « Je te touche avec de l'or cher et honoré poisson, pour que tu deviennes mon familier, je te touche avec de l'or toi ma pêche, sois ma pêche à jamais, multiplie toi cher et honoré poisson, deviens mon familier. "

Les pirogues « à cinq avirons (sur chaque bord)» (lima su avat a cinedkeran) peuvent être comparées aux «maisons à quatre portes" (apat su pantuw a vahei). Elles en ont la grandeur et la magnificence et sont célébrées de la même façon lors de la fin de leur construction. Particulièrement enviées toutes les deux par les "morts errants", les vivants doivent les protéger des nombreux dangers qui les menacent.

Selon les Yami, « une grande pirogue calfatée avec de l'or en poupe est promise à une longue destinée " (amyan su lag na u cinedkeran). «Insubmersible " (jatamehad, jarawa), elle ne craint pas les mers difficiles car elle «ne peut se retourner ». Maintes anecdotes nous comptent comment, lorsque la houle prenait le bateau et menaçait de le faire chavirer, les pêcheurs s'en étaient sortis indemnes car ils avaient de l'or en poupe. "L'or calfaté en poupe » est considéré par les Yami comme une sorte d'assurance sur les biens et sur la vie. Si-Rapungan, à l'exemple de son oncle dont il avait reçu les enseignements et hérité d'un peu d'or, en avait glissé un bout à l'arrière de sa petite pirogue décorée. Il raconte comment une fois, il était parti seul pêcher en petite pirogue.

«Jeune à l'époque, il avait beaucoup de force et pouvait ramer de longues distances sans fatigue. N'ayant pas la moindre touche, il avait poursuivi sa route et ramé si loin outremer (du-ilawud) qu'il ne voyait plus le port et les montagnes de son village. Tout d'un coup, il entendit un grand bruit, le fracas de quelque chose heurtant la coque du bateau avec force, pang! puis encore, pang! cela vint cogner à nouveau. Si-Rapungan regarda à l'intérieur de son bateau et vit une profonde fissure. Avec son pied, il essaya de boucher le trou pour empêcher l'eau de pénétrer mais il n'y eut rien à faire. C'était un énorme requin (rukang) qui revenait à nouveau mordre "l'avant du bateau" (murung nu tatala) et le tirer brusquement. Ouah! c'est un requin faisait le pêcheur tout en essayant d'arrêter l'eau du pied sans succès. Avec une "écope" (sasapsap), il tenta de vider l'eau du bateau, mais l'eau continuait de monter et le requin ne lâchait pas prise. Il attaqua ensuite le bord de la pirogue qui "penchait dangereusement sur le côté" (maligaligan). Si-Rapungan essaya de remettre ses avirons et de ramer comme il pût mais il était poursuivi par le requin qui venait se heurter contre le bateau. C'était une énorme bête, mesurant près de "deux brasses" (adwa adepa), au corps large comme un tronc d'arbre et à la gueule gigantesque et béante. Si-Rapungan était terrifié et essayait de se défendre à l'aide d'un petit couteau et d'un "chant d'exorcisme efficace". Continuant de chanter à l'adresse du requin, il se saisit soudain de ses avirons pour le frapper. Alors le requin s'enfonça peu à peu dans les eaux profondes et disparut. Quel animal terrifiant ! L"âme de gauche" (pahad nu uri) de Si-Rapungan avait eu si peur qu'elle s'était envolée vers un endroit éloigné mais, heureusement, "l'âme de droite" (pahad du wanan) était encore en place. Si-Rapungan avait encore de la force et plus rien à craindre. Il rama lentement tout en bouchant le trou de la coque de son pied et rencontra des hommes en train de "pécher à la senne le poisson volant" (manawar su libangbang). Père-de-Celle-qui-est-féconde (Sian-Mabrak), qui pêchait là plongea voir sous son bateau et ramena un morceau de la "quille qui était cassée" (masyat u rapan). Comme il était dangereux de rester là, dans les parages du requin, tous les pêcheurs prirent la direction du village. Le-Rassembleur (Si-Rapungan) rentra très lentement. De retour sur le port, Grand-père-de-Celle-qui-est-venue- 
d'un-autre-endroit (Siapen-Manliktas) lui demanda ce qu'il était arrivé. Il regarda le bateau et vit que les bords étaient tout "hachurés" (nigetget). C'est vrai qu'il est dangereux de naviguer très loin en haute mer là où les requins sont nombreux. Mais comment un seul requin pouvait-il être arrivé à taillader ainsi des dents de sa mâchoire les bordés du bateau et à en arracher la quille? Que la pirogue soit de cette façon dévastée semblait si étrange que le requin ne pouvait être qu'anitu. Tous ses parents, à leur tour, l'insultèrent lui reprochant d'être allé si loin et par la suite,

il ne retourna plus jamais si loin en haute mer. » requin, mais il se souvient encore de celui de son grand-oncle défunt qui, lui, en rencontra deux. "Ces deux requins avaient vu le bateau de l'ancêtre et le poursuivaient. L'oncle qui était un homme de grande force ramait à toute vitesse afin de les distancer, mais les deux requins continuaient de le suivre de très près. L'homme se fatiguait de ramer et les requins se fatiguaient aussi. Mais les poissons furent bientôt devancés. Arrivé près de La-terre-d'abordage de Du-Irala, il pensa se saisir de son poignard et adressa « un chant efficace » aux requins appelés également « Les poux de Mère-de-Gasyu » (kutu na nina-Gasyu) :

« Aller pêcher dans un endroit très éloigné - Les monts Jangawud et Ji-rakwasangat pour orientation - Attraper de nombreuses dorades coryphènes - Pirogue aux hauts plats-bords rectilignes - À la courbure des projections de poupe et de proue harmonieuse - On t'envie alentour et on nous regarde avec hostilité pour avoir construit un tel bateau ».

Selon le témoignage des pêcheurs, l'or calfaté en poupe de bateau les protège des hostilités des «morts marins » qui essaient, par toutes sortes de moyens, de «pincer les vivants et de faire s'envoler leur âme principale » vers le « domaine des morts » afin de s'emparer de l'embarcation, en particulier si le bateau est neuf et sa construction réussie. Somme toute, l'or «met la chance du côté de la grande pirogue et lui assure une longue vie ainsi qu'aux hommes à son bord» (meilag u cinedkeran ou meilag $u$ pangangapan). Ce précieux calfatage fait de ce bateau " une pirogue riche et pensante " (meinakem a cinedkeran). En yami, le même terme de meinakem a tau désigne à la fois " celui qui a des biens », « celui qui agit avec probité » et " celui qui pense ». La grande pirogue, tel un homme influent du village et un pêcheur expérimenté, "porte sur elle des métaux précieux et a la faculté de penser ». De la sorte, elle saura déjouer les vents qui prennent de la vitesse, les vagues qui se creusent et ne jamais chavirer. Le côté droit de la base de la poupe où est inséré le calfat d'or peut être comparé à l'épaule droite de l'être humain qui est le siège de l'âme principale. « La grande pirogue calfatée à l'or », ou d'« une belle peau en or » (varaten nu piya su kulit a cinedkeran), est composée non seulement d'un corps avec des yeux, des dents, des seins, des cuisses comme tous les bateaux, mais aussi «d'un esprit vital» (amyan su pahad nu cinedkeran) qui la fait entrer dans l'éternité. Insubmersible, « elle est destinée à vivre à jamais dans la lumière du jour » (amyan su lag na du karawan).

\section{Le rituel de lancement}

Les fêtes collectives yami, notamment celles célébrées à la fin de la construction de la maison et le rituel de lancement de la grande pirogue décorée, portent le nom de "nourrir» (meikanekanen) ou de "faire de la nourriture» (mamaring su kanen). La "nourriture de la grande pirogue », composée essentiellement de taros (kanen), a pour fonction de «faire vivre le bateau en l'alimentant». Des taros ordinaires ou «taros à 
entasser " seront d'abord empilés pêle-mêle à l'emplacement de chaque rameur et iront jusqu'à ensevelir le bateau. Puis, des taros de grande taille ou " taros suspendre " (manrangkei su maseve du cinedkeran), auxquels est laissée la partie aérienne, seront posés régulièrement sur l'ensemble et présentés sur des perches de séchage avec des poissons séchés de bon augure telles que les saupes grises ${ }^{22}$. Tous les gens du village viennent aider à la tâche, qui débute parfois à l'aube et se poursuit jusqu'au milieu de l'après-midi. Chaque membre du groupe de pêche doit emplir un espace représentant la "part de nourriture de son aviron ». Les plus riches en tarodières se doivent d'en déposer beaucoup, les autres le font dans la mesure de leurs possibilités. Lorsque tout est bien installé et que le consensus s'établit entre les poseurs de taros, chacun rentre chez soi et fait ses observations sur la superficie de tarodières de chaque pêcheur et sur la grosseur de leurs taros. Le lendemain matin, on commence par enlever les « taros de grande taille» (meiupi, maseve). Chacun ôte ses propres taros, aidé de parents. Les «taros ordinaires » (niman a suli) sont laissés sur le bateau et le partage peut débuter. Après quoi, il en reste souvent.

En « nourrissant la pirogue ", il s'agit pour les « constructeurs" (meicinedkeran a tau) de «nourrir» en conséquence les "gens du village » (makeikeilyan) et les «invités des autres villages" (meinikanikat a kadwan lili). Pour les Yami, un bateau est beau, d'une part si ses bordés supérieurs sont hauts et si la courbure de poupe et de proue est réussie, d'autre part et surtout s'il est bien «nourri ». Plus le bateau croule sous les taros et autres nourritures, plus on dira que c'est un «beau bateau ». L'enclos rituel, situé à côté du bateau et où doivent se serrer en nombre les chèvres et les porcs offerts, participe également en grande part de l'esthétique et provoquera l'admiration de l'assistance, envers le bateau mais aussi envers le "responsable de la pirogue " (mangaharang), qui est l'homme le plus âgé du lignage "constructeur", et de ses " membres » (kakavang du cinedkeran), désormais considérés comme des gens prospères.

Il faut ensuite " alléger le bateau du poids des morts et lui donner la vie ». Après avoir nourri la pirogue et les vivants, on «nourrit les morts " (maparek). De son long couteau à découper, le responsable de la pirogue, paré de son heaume d'argent et de ses ornements d'or, leur offre du jus de canne à sucre, les remerciant de leur aide mais les engageant à quitter au plus vite le bateau et à laisser les navigateurs en vie. Lorsqu'il " entonne le chant d'exorcisme du bateau » (meiavuavuyit), les enfants doivent aller se cacher au loin.

Sur le terre-plein du barreur, l'embarcation présente d'un côté la "poupe orientée vers la terre et la forêt » (maudi du kahasan du teirala) et de l'autre la " proue orientée vers la mer » (murung du teilawud) qui se font face tels deux combattants. De même, l'« homme de la poupe ", le responsable du lignage, est placé à l'arrière tandis que l'« homme de la proue ", représenté par un autre ancien, est placé à l'avant. Ils se font face, le poignard brandi, faisant des gestes d'exorcisme et proférant des insultes, l'un envers l'autre et contre tous les « morts » de l'intérieur du bateau. Le premier à chanter est le plus âgé, à l'arrière, auquel celui de l'avant donne la réplique. Après le " chant d'exorcisme ", les "constructeurs" peuvent «monter à bord pour une célébration par des chants rituels" (meiraud du sahad nu cinedkeran), chacun son tour et selon leur position hiérarchique à l'intérieur du bateau. L'«homme de proue» commence, auquel répondra « celui qui attrape les poissons » (du pangapan su amung), puis les suivants.

Ensuite vient la grande "expulsion des morts du bateau» (mangawui) par tous les hommes du village, surgissant des buissons environnants en file indienne et par 
groupes d'âge. Tout d'abord les "petits garçons" (kanakan) suivis par les «jeunes hommes " (mauva tau) puis les hommes d'âge mûr ou "hommes dressés » (amlida tau), les vétérans «dont la peau se sillonne » (minminyadan su kulit) et enfin «les anciens » (rarake) qui viennent entourer le bateau, les yeux exorbités, tapant des pieds, le poing tournant vivement à hauteur de la poitrine, trépignant de colère, soufflant et criant comme pour «se battre contre le bateau». le but est de «montrer sa force ». Les Yami supposent que les esprits récents du lignage sont venus nombreux, curieux de "voir $»^{23}$ le bateau terminé et d'assister à la cérémonie de lancement. En l'absence de rites d'expulsion, les hommes n'auraient pas la force de soulever leur bateau et de le « lancer dans les airs" (lalabuyin u cinedkeran) comme ils ont coutume de le faire. Lorsque les anitu sont chassés, le bateau devient plus léger. Alors, comme avec un beau jouet, on peut s'amuser et c'est à qui le lancera le plus haut, à qui atteindra la " partie médiane du ciel ». Si par hasard le bateau s'alourdit à nouveau, le responsable de pirogue monte à bord pour donner des coups de couteau et « montrer toute la force de son corps » aux anitu (pangedarten u katautau) qui finissent par ne plus y rester tant cela les effraie. Le bateau, porté à bout de bras est amené sur le port en bord de mer. Il est très beau et très léger et on veut le faire admirer aux gens du village et à ceux des autres villages. Tous, les jeunes comme les vieux, veulent lancer le bateau et le lancer encore pour mieux le faire admirer. C'est un bateau si extraordinaire qu'on l'aime dès qu'on le voit, et on «voudrait bien l'avoir pour soi ». Après un rite d'aspersion d'eau de mer sur la proue du bateau afin qu'elle " teste à jamais droite, rapide et bien orientée ", la pirogue est poussée à l'eau. Lorsque les hommes des autres villages la voient voguer sur les flots, ils la trouvent si belle, si droite et si rapide qu'ils ne peuvent s'empêcher de sortir les armes pour l'attaquer. Comme à un ennemi, ils lui adressent des insultes et des gestes violents afin de lui «montrer leur force » eux aussi, de la combattre et d'essayer de la vaincre pour la ramener chez eux.

\section{Aspersion d'eau de mer et « baptême »}

Avant d'embarquer pour la première fois, le bateau est "aspergé d'eau de mer en tête de proue " (sabuyin u murung nu cinedkeran nu matabwe) par le barreur qui lui adresse cette invocation :

«Proue de notre grande pirogue que j'asperge d'eau de mer, tiens toi toujours haute et droite, file à grande vitesse et sois notre guide à jamais, à nous les hommes et aux poissons. Grande pirogue, donne nous la chance vivre très vieux avec toi, liés que nous sommes à tes entrailles ".

La grande pirogue est ainsi comparée à une femme enceinte qui porte en elle les « marins " (kakavang), les guide et les garde en vie. On asperge de la sorte avec l'eau de source du lignage le sommet de la tête des nouveaux-nés (sabuyin u tuktuk nu matarak a tau) le lendemain de leur naissance afin de les rattacher à leur famille et à leur lignée ancestrale. C'est un rite de longévité et de fertilité destiné à favoriser la bonne santé de l'enfant et à éloigner de lui les maladies (Arnaud 1994). Par cette aspersion rituelle, les pêcheurs cherchent de la même façon à " humaniser » leur bateau, à le rattacher à leur lignée et à prolonger sa vie. La pirogue porte désormais le nom du lignage qui l'a conçue. 


\section{La première sortie en mer et la première pêche}

maison du barreur, deux hommes sortent en mer pour effectuer « la première pêche de la pirogue dans la nuit» (manganyuw nu cinedkeran du maep). Il s'agit "d'offrir en prestation au nouveau bateau le premier poisson pêché » qui a valeur d'augure : Si on pêche la «blennie» sasagiten, c'est elle qui est choisie comme «le premier poisson pêché de la pirogue " (anyuw nu cinedkeran). Il en est de deux sortes dans les eaux du littoral : « la rouge " (mabarangbang a sasagiten) ${ }^{25}$ et « la noire " (mavaeng a sasagiten) ${ }^{26}$ qui sont classées dans la catégorie indigène regroupant des " poissons sans écailles » et des « poissons comestibles par les femmes». Le terme asagiten signifiant aussi «mettre sa ligne à l'eau et attraper immédiatement du poisson ». On considère cette première pêche, par homophonie, comme un heureux présage pour les futures prises du bateau. Si on attrape un "thon ", vauyu ${ }^{27}$, la chance est aussi du côté des pêcheurs : comme la petite blennie, il fait partie « des poissons sans écaille » (vauyu a dimeiisis) considérés comme très proches des hommes. Le "poisson-chèvre" amingan envisagé, par homophonie, comme «faisant rire et donnant de la joie » (tudamying) laisse également " présager une heureuse destinée au bateau » (apiya u lag na, apiya u nilyin na). Enfin la saupe grise, ilek ${ }^{28}$, appelée aussi « la saupe brillante des récifs » (cirayan du rawarawang a ilek), qui est le poisson le plus apprécié des Yami, est là encore un signe très favorable. Le poisson ainsi pêché est ramené à la maison.

\section{Or et navigation à Botel Tobago}

L'histoire de la navigation maritime est liée à la découverte des régions aurifères. Il est difficile de dater les débuts de la métallurgie de l'or (et de l'argent) en Asie du Sud-Est insulaire. Des sites ont été retrouvés à Bornéo : Santubong, exploité de 800 à 1375, et Mindoro aux Philippines, la région sans doute la plus productrice d'or de toute l'Insulinde (Pelras 1989). Bien avant l'arrivée des Européens, les Igorot et les Ifugao récoltaient eux-mêmes le métal pat orpaillage et le travaillaient (Robertson 1914). L'or des Ivatan, au Nord des Philippines, est décrit par Dampier dans ses récits de voyage aux îles Batan et Babuyan (Dampier in Philippines 1683-1690). Il sert à la confection de 
bijoux et constitue l'étalon d'échange prédominant. Le navigateur en évoque la valeur commerciale : «Nous pouvions acheter tous leurs pendants d'oreilles et leurs parures d'or contre quelques barres de fer dont les habitants étaient très demandeurs ». Comme les habitants des îles Batan, les Yami de Botel Tobago savent, depuis des temps très anciens, traiter les métaux et fondre des lamelles d'or et d'argent battus. En 1722, la chronique chinoise ${ }^{29}$ mentionne de l'or et de l'argent en abondance :

«La population, qui semble ignorer l'usage du fer, utilise des métaux précieux pour la confection des têtes de lances et des lames de poignards. Elle fait l'objet de la convoitise de pillards venus de Taiwan dont elle doit repousser les nombreux assauts... »

L'existence de termes apparentés pour désigner «l'or » en yami (vuhawan), dans des langues austronésiennes d'Indonésie, des Philippines ${ }^{30}$ et chez des ethnies des côtes Nord et Est de Taiwan (Ami, Kuvalan, Ketagalan, Siraya) (Kano 1941) indique une connaissance très ancienne du métal et de son traitement, et sans doute son aire de dispersion. D'autre part, la similarité des objets d'autrefois (Asaï 1939) chez les Ivatan, les Itbayat et les Yami, tels que la balance au fléau en forme de pirogue et des réceptacles à poudre d'or en corne de chèvre, laisse supposer des échanges entre ces populations à une époque antérieure au XVII ${ }^{e}$ siècle. Il est possible que les poids de l'ancienne "balance à métaux» yami (panganednan), façonnés dans une variété de quartz inexistante à Botel Tobago, soient originaires des îles Batan (Kano 1941). Le fléau de la balance représente d'autre part dans les deux régions un «bateau " (tatala en yami, tataya en ivatan et en itbayat) : « une petite pirogue » conforme à la réalité dont la hauteur de la proue et de la poupe varie d'une population à l'autre, plus basses chez les Ivatan ou selon l'expression yami « coupées par la moitié ».

Les métaux précieux, l'or et l'argent, sont désignés en yami pat le terme générique de tametamek. Pour traduire le mot " or », on dira vuhawan et le mot "argent ", penrak ou nirpi, du nom des monnaies japonaises. L'or est appelé aussi piya su kulit, «la belle peau ». Il sert à confectionner des plaques en forme de " 8 ", uvei. Assemblées par des liens, elles constituent le pectoral des hommes. L'argent, également très estimé par les Yami, est employé dans la fonte de lamelles servant à fabriquer des bracelets et le heaume. Ce heaume d'argent est une parure imposante aussi prestigieuse que le pectoral d'or conférant à qui le porte toute sa dignité et marquant son rang dans la société. Aujourd'hui, les Yami ne possèdent plus qu'une petite quantité d'or et d'argent qui s'est épuisée au fur et à mesure des partages.

Selon la tradition, c'est l'attrait de l'or qui a conduit les ancêtres des Yami à naviguer vers les îles Batan, notamment Jivatan et Jikbalat où ils auraient procédé à de nombreux échanges. Grand-père-de-Celui-qui-crie-très-fort (Siapen-Jabag du village de Jiraralei) relate comment des ancêtres de son lignage auraient gagné l'île de Jikbalat où ils se seraient liés d'amitié avec des insulaires et auraient échangé des marchandises (tubercules, hameçons, cordages...) contre dix paniers remplis de plaques d'or, de " perles de couleur turquoise » (marapunei) et de gilets cuirassés. Au village de Jiraralei, Sian-Parokso (septembre 1975) a passé la veillée à rappeler le voyage de ses ancêtres de la lignée Du-Chemin (Sira Du-rarahan) par les montagnes et par les îles. L'itinéraire de la fille de Rugrug en particulier, ancêtre fondateur du lignage, une veuve de Jimasik qui prît la mer pour accompagner un Ivatan, Si-Vwang, mène aux îles Batan. Ils reviendront par la suite pour s'établir définitivement au village de Jivalinu. Dans le mythe, les métaux précieux sont souvent acquis illégalement : une jarre remplie d'or et de perles couleur turquoise est dérobée aux Ikardung, une population imaginaire que 
l'éblouissement de la mer suffit à faire mourir. Selon différentes variantes de l'épopée de Siapen-Meiturid, un autre ancêtre du village de Jiratei, les contacts entre les Yami et les Ivatan auraient duré plusieurs années. Si-Vakag, le chef des Ivatan, invita son ami Siapen-Meiturid de nombreuses fois. Considérés tous les deux comme de "grands hommes » à la force herculéenne, il passaient leur temps à se lancer des défis. Mais peu à peu, leurs relations se dégradèrent : un jour, Siapen-Meiturid voulut échanger à SiVakag une grosse pierre couverte de feuille d'or contre de l'or pur. Les Ivatan, jaloux aussi du succès remporté par les Yami auprès de leurs femmes, leur demandèrent de ne plus revenir. Lors d'une dernière équipée, Si-Vakag ordonna aux femmes de danser nues sur la plage pour attifer les Yami. Ceux-ci ne purent résister et approchèrent du rivage où les Ivatan les attaquèrent et tuèrent Siapen-Meiturid et son équipage. Un seul homme revint à Botel Tobago. Selon les listes généalogiques dressées par Kano (1931), cet événement aurait pu avoir lieu vers le milieu du XVII e siècle avant l'arrivée des premiers missionnaires espagnols aux îles Batan.

Les métaux sont considérés aussi comme un don des divinités, appelées les Hommes Du-haut, qui les possèdent. Guluwan, une divinité présentée, par une habitante à un seul et long sein de l'île de Jivuvus (Batan), dont la maison en or brille de mille éclats, fait sortir une plaque d'or gigantesque du sol pour la donner aux hommes représentés par Si-Vwang l'Ivatan et son épouse yami. Mais les hommes sont trop maladroits et l'or est un métal qu'il ne faut pas traiter n'importe comment. Celle qui a tiré dessus sans ménagement voit disparaître la plaque d'or dans un abîme sans fond.

\section{Origine de l'or des pirogues}

Dans la mythologie yami, les excréments de l'aigle pêcheur seraient à l'origine de l'or qui servait lors des rituels de lancement des pirogues. Appelé kangkang en yami, il s'agit d'un "aigle pêcheur massif » avec d'énormes et larges ailes qui a disparu aujourd'hui de Botel Tobago ${ }^{31}$. On dit qu'il y en avait beaucoup autrefois et qu'ils capturaient le poisson en volant bas. Il pouvait prendre des mammifères de la taille d'un chevreau et même des petits enfants.

\section{Variante du mythe de l'Arrière-grand-père Des-abris-à-pirogue (Siapen-Kutan Du-kakamaligan, Jiraralei, 1983)}

« Notre aïeule était rattachée par les femmes au lignage De-bordure-du-village (Sira Du-seili) dont elle descendait en ligne directe. Il faisait beau temps. Notre aïeule prend la jupe qu'elle vient de tisser de bleu indigo. Après l'avoir lavée avec de la cendre, elle va la déposer en bordure de tarodière. Le soleil est au zénith, lorsqu'elle voit un énorme oiseau tournoyer puis fondre sur le vêtement qu'elle vient de laver. Qu'est-ce-que c'est? dit-elle. La première fois où l'oiseau a plongé sur sa jupe, elle peut voir qu'il y a déféqué. Oh là là, mais qu'est-ce-qu'il lui prend à cet oiseau de lâcher ses excréments sur le vêtement que je viens de laver! Elle va voir la jupe qu'elle vient de laver : il y a là des excréments tout tordus comme ceux du crabe valakawan. Mais qu'est-ce-que c'est que cela? dit notre aïeule. Je vais rapporter cela à mon père, dit-elle. Elle enveloppe la chose dans sa jupe et la ramène à la maison.

Une fois rentrée, elle appelle son père : viens voir Papa. Qu'as-tu à me dire mon enfant? Qu'est-ce qui se passe mon enfant? Entre donc Papa, dit-elle. Alors elle ouvre sa jupe pour lui montrer ce qu'il y a à l'intérieur. C'est un énorme oiseau qui a fait ses excréments sur ma jupe. Oui mon enfant, c'est "l'aigle pêcheur" kangkang. Je 
vais inspecter cela de près. C'est de l'or, mon enfant. Comme tu as bien fait de me l'avoir rapporté, mon enfant. Si par hasard tu l'avais jeté, comment l'aurions nous retrouvé ? dit-il. Quand j'ai vu cette chose là, dit la fille, je l'ai tout de suite enveloppée Papa.

Le père fait fondre le métal et se forge des "plaques d'or" (uvei) pour son pectoral. Il martèle également des lamelles plus petites. Puis, afin de montrer leur or et de pouvoir le porter, il organise une grande fête et une large distribution de nourriture. Un cochon gras et deux boucs sont offerts à cette occasion. C'est mon oncle défunt qui m'a rapporté ces faits autrefois. Notre ancêtre à porté son pectoral d'or pour "aller à la rencontre des poissons volants sur le port". Puis il a dit : Si nous fabriquions une grande pirogue. Ils ont commencé à ouvrir des tarodières et à planter des taros. Une multitude de parents sont venus voir les excréments de l'aigle pécheur. Il est extraordinaire cet or, l'or que vous avez trouvé, rapporté chez vous et battu vous-mêmes! Par la suite, les gens sont allés nombreux faire sécher leurs vêtements au soleil. Pourquoi cet oiseau est-t-il venu lâcher ses excréments sur la jupe de notre ancêtre, quelle heureuse destinée avait-elle pour posséder une telle jupe?

Ils construisirent une grande pirogue sur le terre-plein de nos ancêtres du lignage De-bordure où ils organisèrent une grande fête. La part de nourriture de la pirogue constituée de porcs, de chèvres et de taros fût distribuée. »

\section{Variante du mythe d'origine de l'or des pirogues (Mère-de-Tararyu, Sinan-Tararyu, Jiraralei, 1977)}

"Une femme après avoir lavé des vêtements, laissa une jupe usagée par terre pour qu'elle sèche. Quand "l'aigle pêcheur" de grande taille, kankang. vit qu'elle s'éloignait, il plongea sur la jupe où il lâcha ses excréments. Notre ancêtre dit alors : Mais qu'est-ce qui lui prend à cette sale bête, pourquoi est-elle venue déféquer sur mes vêtements? La femme va voir et découvre de l'or appelé "belle peau" ainsi que des "perles de verre" (cinaripan). On sait que les aigles pêcheurs aiment particulièrement manger les petits enfants. Un couple de nos ancêtres n'avait qu'un seul enfant, et l'aigle est venu leur manger leur unique enfant. C'était un garçon dont j'ai oublié le nom, l'un de nos ancêtres, Le père de l'enfant qui avait été mangé prépara ses armes. Il se fabriqua des épieux, un grand poignard et commença à se construire une pirogue. Nos longs colliers et nos parures les plus précieuses nous ont été transmises par notre lignage Des-cocotiers (Sira-Du-Nyui). Si tu étais de notre île, je brandirai mon poignard pour me venger et te fendre le crane de mon épieu. Si je te vois, "aigle-pêcheur", je devrai te tuer car tu as mangé notre enfant, notre seul garçon, et je dois me venger. C'était un grand aigle blanc qui habitait dans un pays éloigné au delà des mers sur une très haute montagne. Dans l'abri de cet aigle blanc de haute montagne, s'amoncelaient toutes sortes de parures étincelantes, des métaux brillants d'or et d'argent et des pierreries de divers colliers. Lorsque notre ancêtre a atteint cette montagne après une année de voyage, il a commencé à l'escalader. Neuf jours plus tard, il montait toujours et n'avait pas atteint son but. Neuf jours encore, il poursuivait sa route et n'avait pas atteint la maison de l'aigle blanc. Onze jours plus tard, il n'avait toujours pas vu le grand aigle blanc. Le mois suivant, à la nouvelle lune, il se trouva en face d'un escarpement rocheux qu'il gravit et où il fit une pause. Peu de temps après il entendit un vrombissement, c'était l'aigle blanc qui revenait de la mer. Il regarda et dans son bec, vit qu'il avait attrapé un petit enfant. L'aigle était en train de manger l'enfant quand l'homme arriva par derrière pour essayer de le transpercer de son poignard. Avec son crochet il voulut l'attraper pour lui donner des coups. Mais l'aigle s'envola entraînant l'homme avec lui. Alors il lui enfonça son poignard dans le coeur et l'oiseau tomba foudroyé. L'homme ouvrit l'aigle blanc de son couteau et de ses viscères en sortirent plein de colliers d'enfant ainsi que des os de petits enfants. L'homme avait apporté avec lui un grand filet de récolte qu'il remplit des parures 
des enfants : plaques d'or, pierreries et colliers. Lorsqu'il redescendit vers la plaine, il rencontra des gens à qui il demanda : vous voyez tous ces colliers d'enfants, si vous reconnaissez les colliers de vos propres enfants, prenez-les! Notre ancêtre s'apprêta ensuite à rentrer chez lui. Et la population pour le remercier lui offrit des présents, des taros de la variété livas, et bien d'autres choses à manger. Quand il rentra chez lui sur l'île de Botel Tobago, tout le monde accourut pour voir les colliers qu'il avait rapportés, ceux du village et ceux des autres villages, tous reprirent les colliers de leurs enfants. »

\section{Récit de son conjoint, Grand-père-de-Nugan (Siapen-Nugan)}

«Il était une ou deux heures de l'après-midi quand un aigle pêcheur se prit les pattes dans la glu. L'aigle s'en dégagea rapidement et s'envola vers Taiwan. Le voilà qui revient vers le mont Ji-Makapapala et notre ancêtre l'aperçoit: comme c'est étrange, dit-il, qu'il tournoie de la sorte au dessus des grottes de Ji-Karaem. L'oiseau a encore de la glu à l'arrière. Comme c'est bizarre dit notre ancêtre. L'oiseau se dirige vers la plaine de Ji-Kanyuyan mais il ne peut revenir. Il tombe à pic dans le goulet de Du-Rawang. Il y a un homme au lieu-dit La-maison-des-poulets. Ji-Vaheuinu-manuk, qui le voit et se précipite. D'autres arrivent en courant de tous les côtés pour voir l'aigle. Même les anciens arrivent à toute vitesse. L'aigle est tombé à DuRawang. Avec des bâtons ils le battent et l'aigle meurt sous les coups. On le sort du goulet. C'est un aigle énorme que l'on porte sur l'épaule à plusieurs à l'aide d'un grosse perche de bois. De retour à la maison, l'un de nos ancêtres va chercher un chevreau, un chevreau qui était avec sa mère pour l'offrir en sacrifice avec l'aigle. Toute la population du village de Jayu s'est ensuite partagé la viande de l'aigle et en a mangé. Le chef de village leur a enseigné la façon de l'attraper, de le préparer et de le manger.

L'aïeul de Grand-père-de-Nugan (Siapen-Nugan) qui avait attrapé l'aigle pêcheur de grande taille a chanté à son intention : "j'ai rêvé à notre source de Du-Jiawang. C'est un grand plateau qui se dresse tel un panier à bijoux. À cet endroit, un aigle blanc y vient souvent. Chaque fois, c'est là-bas qu'il descend. C'est un aigle blanc qui vient de très loin. Un aigle qui peut manger des hommes. Un aigle qui mange des gros boucs et qui mange aussi des porcs gras. Quand il vient sur l'île, il se pose à cet endroit. Cet aigle qui vient de contrées lointaines jusqu'à notre île. Il part outre-mer manger des hommes avec leurs parures, leurs pierres précieuses et leurs bijoux en or". Quand cet aigle blanc revient sur notre île, on l'attrape pour le tuer. Il revient des montagnes d'Outremer et nous l'attrapons pour le transpercer. Parure et métaux d'or ainsi retrouvés sont sortis de ses viscères et passés rituellement au cou des petits enfants qui savent marcher. Si les hommes d'Outremer savaient cela à propos de leurs enfants morts, ils prendraient sûrement leur poignard pour venir nous tuer nous et nos enfants. »

\section{Variante du mythe de l'origine de l'or des pirogues (Grand-père-de- Celui-qui-crie-très-fort, Siapen-jabag, Jiraralei, 1983)}

« Notre ancêtre du lignage Du-chemin (Sira Du-rarahan) était parti en mer pour aller chercher de l'or et des pierreries à suspendre au collier de son enfant qui commençait à marcher ${ }^{32}$. Il était parti très loin au delà des mer et de retour chez lui avertit ses parents : il y a neuf îles et c'est dans la neuvième île que j'ai trouvé "la maison de l'aigle pêcheur". Après l'avoir tué, je suis redescendu vers la plaine me préparant à quitter les lieux. Alors, j'ai rencontré beaucoup d'étrangers qui se tenaient sur leur terre-plein, beaucoup de gens d'outre-mer qui m'ont demandé : "est-ce-que tu l'as tué ? Non, je ne l'ai pas tué, ai-je répondu, car j'ai eu peur de lui". Lui, l'avait tué mais il n'osait pas le dire. Tous ces gens dirent alors: "bien sûr, comment aurait-il pu tuer un aigle aussi énorme, c'est impossible! Tous, dans notre village nous avons essayé une fois ou l'autre de le tuer mais nous n'avons pas pu. 
Toi non plus. Comment aurais-tu pu le tuer? Pourtant, dit notre ancêtre, l'homme de notre île : je l'ai tué cet aigle et bien tué. Comment a-t-il été tué ? L'aigle était à l'intérieur d'une maison d'habitation près du poteau principal en train de manger un petit enfant. Je suis rentré tout doucement dans la maison, j'ai levé mon pilon et je l'ai abattu sur lui avec force. Mais je dois partir maintenant. Lorsque j'ai tué cet aigle, dit notre homme, j'avais une raison profonde : je connaissais votre chef de village et je m'étais lié d'amitié avec lui". Il resta là quelques jours dans leur maison et le chef dit aux gens du village: "cet homme a tué notre aigle, c'est extraordinaire. Nous devons le récompenser pour son geste. Quand on voyait cet aigle pécheur gigantesque, que ce soient les enfants ou les adultes, nous devions nous cacher en vitesse dans les hautes herbes du bord du chemin. Il fallait trouver n'importe quel endroit pour se cacher. Il était alors impossible de travailler. Quand on voyait l'aigle, il pouvait à tout moment nous manger. Nous ne pouvions plus aller à la montagne. Nous ne pouvions plus non plus rester tranquillement assis à l'extérieur car nous avions peur que l'aigle ne vienne nous manger".

Ces gens discutent entre eux de la question. Puis tous les villageois viennent lui donner quelque chose. Il y en a qui apportent des poulets. Il y a tant et tant de présents qu'ils en font un grand tas au bord de la mer. "Pardon, dit notre homme de l'île mais vous m'avez donné trop d'affaires et je ne peux pas tout ramener. Si je regarde les choses que vous m'avez données, cela me suffit. Ce que je pourrai ramener, je le ramènerai, mais le reste je me contenterai de le regarder. Mon bateau est trop petit pour ramener le tout. Il ne peut contenir toutes ces choses". Il met ce qu'il peut dans son bateau et tous les gens du village viennent l'aident à pousser sa pirogue pour repartir. Les amis se disent au revoir.

Puis, notre ancêtre rencontre une autre île: "d'où viens-tu? Pourquoi es-tu ainsi chargé ? Il leur dit : ces choses viennent d'une autre île ; on me les a données car j'ai tué l'aigle pêcheur. La population était heureuse et elle m'a fait cadeau de tous ces présents. Est-ce-que cela a une importance pour vous autres? Tout le monde t'en est très reconnaissant. Il n'y avait plus moyen de sortir à l'extérieur de peur que l'aigle ne vienne nous manger. Maintenant que l'on sait que tu l'as tué, on peut sortir en toute liberté". Ceux-là lui donnent aussi des présents et il choisit les plus beaux car il n'avait pas la possibilité de tout prendre. Il y avait trop de choses et le bateau aurait été immobilisé.

Puis, il poursuit son chemin et rencontre une autre île où on lui donne encore des présents. Et ainsi de suite, tout au long de son voyage par différentes îles vers Botel Tobago. S'orientant vers le mont Jipanunugatan, il guide son embarcation dans cette direction. Arrivé près du rivage, il pense : toutes ces choses, je ne vais pas les ramener jusqu'au port car tous ces vêtements, toutes ces choses que ces gens m'ont donnés sont porteurs de souillures et de maladies. Aussi vais-je m'arrêter au récif, appelé La-maison-des-poulets, Ji-vahei-nu-manuk. Notre ancêtre descend là où il construit un poulailler. Aussi ce récif s'est-il appelé La-maison-des-poulets car c'est là que notre ancêtre a laissé ses poulets. Au récif de Ji-pangarikan, Là-où-on-faitsécher-des-vêtements, il met à sécher tous les vêtement qu'on lui a donnés, d'où le nom du récif. A cet endroit, il a laissé toutes les choses qui pesaient trop lourd et n'a gardé pour lui que le meilleur, l'or et les pierreries qu'il a ramenés chez lui. C'est ce que j'ai entendu dire de mon grand-père, je ne connais pas la suite de l'histoire. Les vêtements qu'il avait rapportés de pays lointains, il ne les a pas donnés aux habitants de sa maison, car il craignait qu'ils ne leur transmettent des souillures et des maladies. Il a laissé les vêtements au Récif-où-sèchent-les-vêtements, à DuPangarikan. Lorsqu'il est rentré chez lui, il a partagé les objets qu'il avait ramenés avec chacun de ses parents. C'est ainsi que notre ancêtre a "passé le collier au cou de son enfant", puis a décidé de construire un bateau, une grande pirogue qu'il a calfaté avec de l'or des excréments d'aigle pécheur rapporté des pays d'outre-mer. " sociale et symbolique d'un petit enfant jusqu'à ce qu'il soit autonome (Arnaud 1993). 
Elle s'étale sur la même période de temps, deux ans depuis sa conception jusqu'à l'âge de marcher. Comme celle de "l'enfant-esprit », la création d'un bateau est présidée par les puissances surnaturelles. La décision de construire une pirogue et par là-même de former un futur groupe de pêche est prise sous les auspices des divinités du Haut ou des Pères-du-Haut lors du grand appel rituel des poissons volants sur le port. Comme les patents soumis à de nombreux rites et interdits dont dépend la survie du fotus puis du nouveau-né, le futur groupe de pêche participe intensément à l'élaboration de son bateau. Ils deviennent "les constructeurs" ou «les travailleurs»; de leur force de travail, de l'observation des rites dédiés aux différentes pièces de bois au fur et à mesure de l'assemblage des bordés, de la quantité d'offrandes apportées en prestation, etc. dépend toute l'espérance de vie du bateau.

Les bûcherons tenteront de " durcir " leur bateau tout en "se durcissant eux-mêmes " au fur et à mesure du façonnage des planches. Ils déjoueront les pièges des «morts » (anitu) qui cherchent à les affaiblir, par toutes sortes de moyens, pour s'emparer de l'embarcation. À cet effet et pour "fortifier leur bateau », ils utiliseront le bois d'œuvre «d'arbres forts », marqueront de sang et apposeront du métal d'or sur la quille, la poupe et la proue où viendront s'assembler tous les autres bordés... Peu à peu, «les travailleurs donneront la vie à leur grande pirogue ». Lors de son lancement enfin, plus "ses cuisses seront rebondies de taros ", plus « elle recevra de nourriture ", meilleure sera sa santé. La «fabrication d'une grande pirogue " n'est finalisée qu'avec «son calfatage d'or » qui décide de son avenir. Ainsi "calfatée ", elle acquiert « un esprit vital » et semble «s'humaniser ». Elle devient autonome et « insubmersible, destinée à voguer éternellement dans la clarté ».

51 Mais pour quelle durée vogueront encore les grandes pirogues de Botel Tobago? Désormais, les "groupes détenteurs de filets" ont tendance à remplacer progressivement les "groupes de pêche en grande pirogue ». Les pêcheurs utilisent toujours leurs grandes pirogues mais n'en construisent plus de nouvelles. Elles sont remplacées peu à peu par des bateaux à moteur achetés par de jeunes Yami à Taiwan. Certains construisent encore des petites pirogues pour leur usage personnel et pour la pêche collective à la senne. Tous les villageois se cotisent aujourd'hui (1999) pour acheter des filets en commun. Chaque Iraralei ou presque a sa part « de grande senne » dans "un groupe détenteur de filet» (asa pananawaran). Il y a ainsi plusieurs grands groupes à Jiraralei, composés de parents mais aussi de cousins éloignés, d'amis etc. qui pêchent ensemble et s'en partagent le produit. Les techniques de pêche ont évolué également. Les mois de mai et de juin qui étaient consacrés autrefois à la pêche à la ligne de traîne en petite pirogue sont voués principalement de nos jours à la pêche aux grands filets dont la taille et le nombre permettent de rapporter des prises plus abondantes que par le passé. 


\section{BIBLIOGRAPHIE}

Asaï, Erin, 1939, « An ethnographical link between the Batanese and the Yami », Nanpo Dozoku V

(3-4) : 73-77 (Tokyo).

Arnaud, Véronique, 1979, "Cohabitation difficile entre les Yami et les âmes de leurs morts », Asie du Sud-Est et Monde Austronésien (ASEMI)X(2) : 119-169).

- 1984, Les Hommes de l'île de Botel Tobago. Histoire d'un peuple sans écriture et espace villageois. Thèse de Doctorat d'État es Lettres et Sciences Humaines, sous la direction de G. Condominas, Paris.

- 1994, «L'enfant-esprit. La naissance chez les Yami de Botel Tobago », pp. 9-36, in J. Koubi et J. Massard-Vincent (eds), Enfants et Sociétés d'Asie du Sud-Est. Paris : L'Harmattan.

- 1995, « Les grandes pirogues des Yami de Botel Tobago », Anthropologie Maritime, Les hommes et les bateaux. Usages, appropriation et représentation 5 : 79-95.

- 1999, « Longévité et ancêtres (Botel Tobago, Taiwan) », pp. 61-83, in J. Massard-Vincent et S. Pauwels (eds), D'un nom à l'autre en Asie du Sud-Est, Approches ethnologiques. Paris : Karthala.

Chen Chi-lu, 1968, Material Culture of the Formosan Aborigenes. Taipei : The Taiwan Museum.

Dampier in Philippines

1683-90, Manila, The Philippine Islands, vol.39.

Dezsö, Benedek

- 1991, The songs of the Ancestors. A comparative Study of Bashiic Folklore.

Taipei : South Material Center (SMC) Publishing INC.

Fox, C. E.

- 1908, The threshold of the Pacific. New York : Alfred A. Knopf.

Kano, Tadao, 1931, « Notes on the Boat among the Yami tribe in Botel Tobago

(Formosa) », The Journal of the Anthropological Society of Tokyo XLVI, 525 : 262-272 (en japonais). - 1938, «Boat Building and its ceremony among the Yami », The Journal of the Anthropological Society of Tokyo, LIII, $606: 125-146$ (en japonais).

- 1941, " Gold Culture among the Aborigenes of the Philippines and Formosa ", The Journal of the Anthropological Society of Tokyo LVI, 646 : 465-476 (en japonais).

Pelras, Christian, 1989, « Métal et métallurgie en Insulinde », in H. Schubnel (ed.), Métal, Hommes et Dieux. Paris : Muséum National d'Histoire Naturelle.

Robertson, J.A., 1914, « The Igorots of Lepanto », Philipine Journal of Sciences IX, D, $6: 465-530$.

Yamada, Yukihiro, 1966, A Preliminary Itbayaten Vocabulary. University of the Philippines : Institute of Asian Studies.

- 1967, « Fishing Economy of the Itbayat, Batanes, Philippines, with Special Reference to its Vocabulary ", Asian Studies 5, 1.

\section{NOTES}

2. Cette période est divisée en quatre lunaisons : kapuan, « dixième lunaison » (mars) ; peikaukaud, « lunaison des avirons » (avril) ; papataw, « lunaison de pêche de jour à la traîne » (mai) ; pipilapila, « lunaison d'or» (juin).

3. J'ai décrit les bateaux yami et leur organisation dans ma thèse (1984). 
4. Ces couleurs étaient autrefois obtenues avec de l'argile, de la suie et de la chaux des bénitiers.

5. Dans une étude sur l'économie de la pêche à Itbayat, Yamada (1966) répertorie différentes embarcations dont le tataya, terme générique pour les bateaux et l'avang. employé aussi en yami pour désigner autrefois de grands bateaux destinés aux longs voyages, armés de quinze avirons sur chaque bord et d'une capacité de trente rameurs. Ce mot s'applique de nos jours aux grands navires étrangers.

6. Zanthoxylum integrifolium. Merrill. Rutaceae.

7. Selon W. White du département de Géosciences de l'Université de Pennsylavanie (in Dezsö 1991), ces perles opaques de verre bleu pourraient être d'origine chinoise et dater d'il y a sept cent ou mille ans. Les Yami pensent que, comme l'or, elles sont originaires des iles Batan où les habitants les trouvaient enfouies dans le sol et avaient coutume, à la manière des Yami, de les jeter à la mer lors du premier jour de pêche des poissons volants.

8. Dans la pensée yami, l'or comme les perles de verre bleues sont associés au sang qu'ils ont le pouvoir de coaguler. Si deux hommes se battent et qu'il y a un blessé, le coupable est celui qui a causé la blessure ; ce dernier est tenu de « rembourser (la blessure)» (meitumuru) de sa victime par une lamelle d'or de la taille de la plaie -ou quelques perles bleues- représentant « la part de nourriture de la blessure » qui est appliquée sur la lésion. La même prestation est requise pour des délits d'adultère.

9. Cette pratique est également associée aux rites de fertilité.

10. Selon Kano (1938), les hommes qui participent pour la première fois à la construction d'un grand bateau sont soumis à certains interdits : ainsi ne doivent-ils pas consommer de poissons ni de fruits frais de peur de voir, comme ces aliments dont la peau se détache, leurs membres ou leurs fesses peler au cours de la navigation.

11. Il est par exemple interdit de collecter des coquillages sur le rivage, de pratiquer la pêche à la ligne ou sous-marine pendant la saison de pêche.

12. Uuyat, en yami, qui désigne à la fois les - veines dures et saillantes » de l'homme et du bois, porte aussi le sens de la «force ». La «force d'un seul homme », «le travail d'un seul homme » pour son bateau (ou sa maison) face à des « morts » omniprésents dans la nature.

13. Ilek, Kyphosus cinerascens, Forskäl.

14. Cayi, acayi. Pometia Pinnata. Forster, Sapindacée.

15. Cipuwu, Artocarpus lanceolata. Trec., Moracée.

16. Maracyayi, Chisocheton kanehirai, Sasaki, Méliacée - Anungu. Ficus auriculata. Lour. MoraceeIsis. Dracontomelum colule. Blanco-Skells. Anacardiacée.

17. Itap, Neonauclea reticulata. Merrill. Rubiacée.

18. Tels que les arbres poro (Michelia compressa (Maxim.). Sarg., var. formosans, Kaneh. Magnoliacée) -vusingsing (Astronia cumingiana. (Vidal), Kaneh. Melastomacée) - et Panguwen (Eugenia cumingiana. Vidal, Myrtacée).

19. Pabwebwesen su piya su kulit = tuytuynen : «appliquer de l'or », désigné sous le nom de «belle peau ", de façon rituelle, sur la nouvelle construction.

20. Seuls les arrières-grands-parents ayant des arrières-petits-fils et portant le nom de SiapenKutan peuvent célébrer ce rituel (Arnaud 1999).

21. Littéralement des « arcs-en-ciel de mer».

22. Suspendre des poissons (mangamung) sur la grande pirogue lors du rituel de fin de construction n'est autorisé que pour ceux qui sont reconnus comme de bons pécheurs.

23. Bien qu'ils soient décrits comme dépourvus d'yeux.

24. Ces chants meikarusan, différent d'un village à l'autre, peuvent aussi être exécutés de retour d'une pêche fructueuse lorsque les pêcheurs sont joyeux et rament à grande vitesse pour faire preuve de leur force.

25. Entomacrodus decussatus. Bleniidae (Bleeker).

26. Salarias fasciatus, Bleniidae (Bloch). 
27. Thunnus thynnus, Scombridae (Linnaeus) - Gymnosarda unicolor, Scombridae (Rüppel).

28. Kyphosus cinerascens, Girellidae (Forsskal).

29. Fengsu liu kao (Six études sur la coutume), 1722.

30. Lexique Thématique Plurilingue de trente-six langues et dialectes d'Asie du Sud-Est insulaire (1997:

81).

31. Il existe un rapace similaire de taille plus petite, «l'aigle pêcheur » manayi (ou pygargue à queue blanche, Haliaetus albicilla).

32. Rituel du « passage du collier » (maparaka) destiné à l'enfant qui commence à marcher.

\section{RÉSUMÉS}

La construction d'une grande pirogue suit un long processus et comporte trois phases: la préparation qui s'étale sur une période d'environ trois ans jusqu 'à la maturité des taros, la fabrication qui se définit comme «le travail » accompli par les membres du groupe de pêche qui unissent leur force à cet effet, et le rituel de lancement où l'on se propose de «nourrir » le bateau par des rites propitiatoires dédiés aux différentes pièces de bois, au cours desquels on calfate avec l'or, on pratique des onctions de sang, des chants et des gestes d'exorcisme, qui contribuent à «donner peu à peu la vie au bateau ». De l'or calfaté entre les joints de la poupe et de la quille décide de son avenir.

A long process is necessary in order to build a large pirogue; it includes three phases: the preparation that takes place over a period of three years before the taro plants reach their maturity; the work accomplished by the members of the fishing group who unite their forces to this end, and the ritual of launching, in order to "nourish" the boat using propitiatory rites dedicated to different pieces of wood during which gold is used for caulking and blood unctuosis are practised; chants and exorcist gestures are made that serve to give, little by little, life to the boat. The caulked gold between the joints of the prow and the keel will be a determining factor in the the destiny of the boat.

La constucción de una gran piragua sigue un largo proceso y comporta tres faces : la preparación que se extiende por un período de alrededor de tres años hasta la maduración de los taros, la fabricación que se define como « el trabajo » cumplido por los miembros del grupo de pescadores, quienes unen sus fuerzas con ese fin, y el ritual del lanzamiento, donde se propone «nutrir » el barco por medio de ritos propiciatorios dedicados a las distintas piezas de madera, durante el curso de las cuales se calfatea con oro, se practican unciones de sangre, cantos y gestos de exorcismo, los cuales contribuyen a «dar poco a poco la vida al barco ». Del oro calfateado entre las juntas de la popa y de la quilla depende su porvenir.

\section{INDEX}

Mots-clés : grande pirogue, or, rituels, Taiwan, Yami 
AUTEUR

VÉRONIQUE ARNAUD

CNRS, UPR 297, LASEMA, Centre A.-G. Haudricourt, 7 rue Guy Môquet, 94801 Villejuif Cedex 